\title{
CRITERIOS DE SELECCIÓN DE LOS CONTENIDOS DEL CURRÍCULUM
}

\section{Selection criteria of curriculum contents}

Julio VERA VILA, Ángel GARCÍA DEL DUJO, José Vicente PEÑA CALVO y Bernardo GARGALLO LÓPEZ Universidad de Málaga. Departamento de Teoria e Historia de la Educación. Facultad de Ciencias de la Educación. Campus de Teatinos. 29071 Málaga. Universidad de Salamanca. Departamento de Teoría e Historia de la Educación. Facultad de Educación. Paseo de Canalejas, 169. 37008 Salamanca. Universidad de Oviedo. Departamento de Ciencias de la Educación. Facultad de Filosofía y Ciencias de la Educación. Aniceto Sela, s/n., 33005 Oviedo. Universidad de Valencia. Departamento de Teoria de la Educación. Facultad de Filosofía y Ciencias de la Educación. Blasco Ibáñez, 21. 46010 Valencia.

Fecha de aceptación definitiva: marzo de 2000.

BIBLID [(1130-3743) 11, 1999, 13-52]

RESUMEN

En un momento en el que la información se produce en grandes cantidades, por medios muy diversos y a una velocidad muy grande, uno de los problemas pedagógicos más importantes es el de establecer cuáles son los contenidos básicos que merece la pena aprender. Este trabajo se centra en la cuestión previa de ofrecer criterios para establecer prioridades a la hora de seleccionarlos, teniendo en cuenta que en esta tarea influyen los demás elementos del diseño curricular, especialmente los objetivos y los procesos de aprendizaje.

SUMMARY

In a moment in which most information is produced by different ways and so quickly, one of the most important paedagogical problems is to stablish which are the basic contents that must be learnt. This work is focused on the previous question 
of offering criteria for stablishing priorities in order to select them, considering that in this matter the other elements of curriculum design have also influence, especially the points and processes of learning.

\section{LA SELECCIÓN DE LOS CONTENIDOS COMO PROBLEMA PEDAGÓGICO}

Este trabajo aborda un tema tan complejo como es el de los criterios de selección de los contenidos del currículum. Nuestro objetivo ha sido poner en evidencia la necesidad de hacer algún tipo de selección y explicitar los muchos problemas que conlleva esa tarea. Finalmente, todas sus páginas han sido escritas con el propósito de señalar los criterios que hoy aparecen como los más razonables, dando por sentado que no son los únicos posibles y que sólo pretenden servir de elementos para la reflexión. Los criterios son requisitos o condiciones que se proponen para establecer un orden de prioridad entre los contenidos, pero no son propiamente ni contenidos, ni condiciones de aprendizaje, ni métodos de enseñanza, aunque los implican a todos ellos.

Desde luego, el tema no es nuevo; tiene una larga tradición y ha sido objeto de atención desde las más diversas perspectivas: filosófica, didáctica, epistemológica, política, sociológica, psicológica, etc. En concreto, este tema ha sido abordado en varias ocasiones en los seminarios y congresos de Teoría de la Educación dando lugar a publicaciones que se recogen en la bibliografía. Aquí se ha pretendido dar una visión pedagógica que entiende que los contenidos son un elemento integrado en un sistema de factores que conforman el diseño curricular como un instrumento, un recurso o una ayuda para la práctica de la enseñanza que realiza cada profesor o equipo de profesores en su centro.

En realidad los contenidos propuestos en un diseño sólo adquieren virtualidad en la práctica real de los profesores. Por ello, ningún esfuerzo de sistematización pedagógica del currículum podrá tener reflejo en las instituciones educativas, si no tiene ningún efecto en la etapa final de la selección de los contenidos, es decir, en la evaluación. Y es que el significado de los contenidos se desvirtúa demasiadas veces, convirtiéndose sólo en requisitos transitorios para pasar de un curso a otro dentro de los diferentes niveles del sistema educativo. Cuando esto ocurre, el alumno aprende de forma relevante sólo aquello que considera necesario para sobrevivir con éxito a las exigencias de la cultura escolar, lo cual viene determinado, en gran parte, por el sistema de evaluación.

Por ello, en un momento como el actual en el que están en revisión los contenidos escolares, sería bueno que pensáramos no sólo desde la lógica de las materias, sino también y principalmente, desde las necesidades y procesos de aprendizaje de los alumnos, por una parte, y desde un criterio de utilidad de los contenidos a la hora de ayudar a comprender y valorar la realidad, por otra. Todo ello es difícil con el sistema actual de formación inicial del profesorado, claramente descompensado a favor del dominio de la materia y en detrimento de los componentes pedagógi- 
cos, lo que da lugar a que muchos profesores no hayan tenido apenas experiencias en la utilización del conocimiento como recurso para el aprendizaje o simplemente en su transmisión. Sin un cambio en la formación de los profesores que potencie la reflexión sobre el componente pedagógico de la enseñanza, es decir, lo que se hace y cómo se hace con el objeto de conocimiento para crear contextos que favorezcan el aprendizaje, será muy difícil crear una cultura profesional que potencie la interdisciplinariedad y el trabajo en equipo, que son factores básicos en los procesos reales de selección, organización, forma de transmisión y evaluación de los contenidos.

\subsection{La necesidad de seleccionar los contenidos}

En el momento actual, la información se produce en tales cantidades, por medios tan diversos y a una velocidad tan grande, que el problema es cómo seleccionarla, asimilarla y controlarla al servicio de objetivos valiosos al mismo ritmo en que se produce. El modelo humanista del saber necesita una reelaboración acorde con las circunstancias actuales. Ni se puede saber todo, ni seguramente merece la pena; por ello, determinar cuáles son los aprendizajes que merece la pena adquirir a lo largo de la vida ante esta realidad es uno de los dilemas pedagógicos más difíciles de resolver.

Uno de los intelectuales españoles que con más sincera radicalidad se planteó, al comienzo de los años treinta, el problema de la sobreproducción de información fue Ortega y Gasset. Es soprendente cómo sus reflexiones, dirigidas entonces a la necesidad de reformar la enseñanza universitaria, cobran pleno sentido hoy, si las dirigimos a la enseñanza básica. Su reflexión parte de la constatación de que la vida del hombre es una selva laberíntica que exige un grado de cultura general suficiente, un sistema ordenado y claro de las ideas sobre el mundo y la humanidad, como para poder trazarse planes razonables. Esa cultura necesaria para todo hombre es lo contrario de la erudición o el ornamento (Ortega, 1975, pp. 39 y 45).

La cultura general que defiende es una síntesis sistematizada de los saberes que nos reportan una idea del mundo y del hombre, a partir de la cual nos es más fácil entendernos, reflexionar críticamente, conducirnos en la vida y seguir aprendiendo. Por ello nos advierte que "ha llegado a ser un asunto urgentísimo e inexcusable de la humanidad inventar una técnica para vérselas adecuadamente con la acumulación de saber que hoy posee. Si no encuentra maneras fáciles para dominar esa vegetación exuberante quedará el hombre ahogado por ella" (Ortega, 1975, p. 79).

Mucho más recientemente Edgar Morin se queja de que la excesiva fragmentación de los objetos de estudio y del saber ha dado lugar a una inteligencia ciega. "La ciencia se ha vuelto ciega por su incapacidad de controlar, prever, incluso concebir su rol social, por su incapacidad de integrar, articular, reflexionar sus propios conocimientos" (1994, p. 79). Por ello reclama una lógica de la complejidad de lo que está tejido en conjunto, de lo uno y lo múltiple. 
En una línea muy similar, Postman (1994) afirma que la contribución mas importante que pueden hacer las escuelas a la educación es la de encontrar un núcleo moral, social e intelectual que dote de un sentido de coherencia, de un sentido de finalidad, a sus estudios. Por ello propone como principio integrador, como fundamento de la educación, la cultura entendida como "la búsqueda de una comprensión unificada de la naturaleza y de nuestro lugar en ella" (p. 240). Su propuesta se centra en aprender lo más esencial de la historia de la humanidad (arte, ciencia, religión, lenguajes, ética, etc.) bajo un principio de coherencia que exprese la continuidad abierta de la iniciativa humana. En definitiva, como señala Vázquez (1999, p. 32) la primera tarea de todo programa educativo es la de "facilitar la indagación del hombre acerca del propio hombre" y en ese sentido cabe defender la primacía de la orientación humanística del currículum.

Como cada vez se produce más información en menos tiempo, por una lógica simplista, es decir, muy eficaz, los expertos en cada materia tienen la tendencia a querer incluir todos esos datos, teorías y procedimientos en los currícula. El resultado es: a) que el crecimiento de los datos no viene acompañado de una nueva síntesis del campo en cuestión desde una perspectiva actual y b) que a medida que crecen los datos a aprender aumenta el peso de los procesos de memorización y decrece (a jornada escolar igual) el de los procesos cognitivos más relevantes como la síntesis, el análisis o la evaluación, y no digamos nada el de los procedimientos y las actitudes (Esteve; Vera; Terrón; Franco y Civila, 1998). Pues bien, hay que tener claro que lo que es de gran valor para una disciplina, en términos científicos o profesionales, no lo es automáticamente para el aprendizaje educativo que ha de articular de forma coherente los diversos elementos del diseño curricular para propiciar el desarrollo integral de la persona. La información sólo es útil para quien tiene una razón desarrollada, porque el conocimiento es reflexión sobre la información (Savater, 1999, p. 59). Por eso la educación no puede ser transmisión de información. Cuando alguien nos da informaciones ordenadas, relacionadas, unidas bajo algún principio de coherencia temática y pedagógica, nos da algo más que información.

Los principios de economía de la enseñanza y de coherencia del currículum coinciden, pues, como vamos viendo, en una búsqueda coherente e integrada de los fundamentos en la historia de la cultura humana. Esto merece algunas reflexiones adicionales:

a) Esa perspectiva no sólo no debe prescindir de los conflictos que han tenido lugar en la historia de las ideas, sino que son necesarios para comprender realmente sus aspectos afectivos, emocionales, los choques entre creencias, de donde como decía Ortega, surgen las ideas. Es la postura que defienden autores como Young, Bernstein, Lundgren, Apple, Popkewitz, etc.

b) La búsqueda de las raíces históricas para comprender el presente debe contemplar los dos términos de la ecuación, esto es, el pasado y el presente. Ello quiere decir que es esencial encontrar en el conocimiento de los gran- 
des problemas del presente las razones para revisar sus orígenes. Lo que estamos proponiendo es partir de la realidad actual y de la cultura próxima del alumno, pero contextualizándolas en la aventura histórica del hombre (postura reconceptualista).

c) En tercer lugar, la gran ventaja de este enfoque de los contenidos es que libera tiempo para aprender. Ortega decía con deslumbrante ironía que lo que nos ahorramos en el enseñar lo ganamos en cantidad de aprendizaje efectivo (Ortega, p. 76). Lo que se quiere decir es que aprender cosas esenciales, precisamente porque representan una síntesis creativa de algo más específico, requiere tiempo, dedicación y sosiego.

d) Por último, se evidencia que no basta seleccionar los contenidos, sino que es de vital importancia la forma en la que sean enseñados y aprendidos. Aunque fuéramos capaces de llegar a algún acuerdo respecto a lo que es fundamental aprender hoy, en las instituciones educativas, todavía nos quedaría por imbuir de esos mismos principios a la forma de enseñarlos, a los métodos, a las actitudes con que se enseñan y a la forma de evaluarlos. De lo contrario, se corre el riesgo de volver a las antiguas enciclopedias, que eran meros resúmenes de datos inertes.

Cuanto llevamos dicho podría resumirse en dos principios generales de selección de los contenidos que podemos tener en cuenta:

a) Principio de economía de la enseñanza o de búsqueda de integración sistémica. Significa comprender que no podemos, ni merece la pena, saberlo todo, experimentarlo todo, sentirlo todo. Que tanto la realidad como cada persona son una unidad. La realidad debe estudiarse buscando esquemas comprensivos integradores (Colom, 1987; Torres, 1994), y la persona que aprende ha de ser entendida como una interrelación de aspectos afectivos, morales, cognitivos, fisiológicos, artísticos, etc. La pedagogía es en gran parte un saber selectivo e integrador que reelabora desde sus propias exigencias las aportaciones de los más diferentes campos; por ello es fundamental en la formación profesores para ayudarles a cambiar su cultura profesional.

b) Principio de coherencia. Todo el proceso educativo necesita un núcleo moral, social e intelectual que dé sentido y coherencia a cada uno de los aprendizajes. Ese núcleo puede consistir en comprender el aprendizaje educativo como una inmersión en la aventura por comprender el mundo y nuestro lugar en él. Este núcleo se postula como el organizador previo por antonomasia capaz de dar sentido y aglutinar todos los contenidos escolares. 


\subsection{El concepto de contenido y sus tipos}

Una de las cuestiones fundamentales que debemos afrontar es la de determinar a qué tipo de realidades nos referimos al hablar de contenidos del currículo. Como indica Sarramona (1989, p. 197), los contenidos tradicionales han sido los conocimientos vinculados a las disciplinas académicas; sin embargo, hoy suele usarse este concepto en un sentido más amplio y a la vez más rico. Así, por ejemplo, A. Pérez (1988, p. 117) considera que junto a los conocimientos deben incluirse el arte y la moral, ya que todas ellas conforman la realidad material y mental en la que se desenvuelve el sujeto que aprende.

De la misma manera, la reforma del sistema educativo español concede gran importancia a los contenidos, pero lo hace de una forma diferente al tratamiento que habían recibido tradicionalmente. Concretamente se entiende que los contenidos escolares son una selección de elementos culturales en un sentido antropológico tan amplio que incluye conceptos, lenguajes, valores, sentimientos, actitudes, creencias, habilidades, procedimientos, pautas de comportamiento, etc. (Coll, 1992, p. 14). Al entenderlos de esta manera, intenta superar ciertas concepciones basadas casi exclusivamente en el "Saber qué", para señalar la necesidad de abordar otros aprendizajes como el "saber por qué", el "saber cómo", el "saber para qué", el "saber hacer", el "querer saber, querer hacer, querer sentir, querer experimentar, querer compartir", etc. El problema, cuando se adopta un concepto tan amplio, es que se produce una cierta confusión entre los contenidos y otros elementos del diseño curricular como los objetivos y las actividades fundamentalmente.

A fin de concretar el uso del término y reducir la posibilidad de confusión con otros elementos del diseño curricular, consideraremos que los contenidos son un conjunto de saberes y productos culturales seleccionados para ser aprendidos por los alumnos que responden al interrogante de "qué" hay que enseñar/aprender, por lo que abarcan conocimientos (hechos, conceptos y principios), procedimientos, normas y valores. Esta es la propuesta del profesor Sarramona (1999, p. 85), a partir de la cual plantea la posibilidad de alcanzar cualquier tipo de objetivo ya esté relacionado con la adquisición de conocimientos, de habilidades o de actitudes. Aunque los documentos de la Logse incluyen también las actitudes de los alumnos, consideramos que es mejor no confundirlas con los contenidos, ya que, estrictamente hablando, se trata de objetivos a alcanzar o de procesos a desarrollar.

No obstante, la distinción entre contenidos explícitos y contenidos implícitos u ocultos puede servirnos para clarificar algo más esta cuestión. Los primeros son productos culturales elaborados por la humanidad a lo largo de la historia y que, por lo tanto, son objetivables aunque revisables y criticables. Esos productos culturales incluyen hechos, conceptos, teorías, procedimientos, sistemas y criterios de valoración, normas, y también es posible contemplar los planteamientos ideológicos y actitudinales de los sujetos que protagonizaron los hechos a analizar como contenidos educativos. Los contenidos implícitos, por su parte, son aquellos repertorios de conocimientos, procedimientos, normas y valores que la cultura del centro en su 
conjunto, aunque más directamente los profesores, transmiten de forma asistemática, no planificada e inconsciente a los alumnos. Tanto unos como otros representan un conjunto de oportunidades de aprendizaje que la institución ofrece para el desarrollo pleno de la personalidad y no sólo en su faceta intelectual o racionalista.

Este interés por el desarrollo del componente comportamental y actitudinal del sujeto es precisamente el que ha llevado a incluir, en los documentos de la Logse, las actitudes como un tipo de contenido más, cuando el desarrollo deseable de éstas es, más bien, un objetivo o un resultado a alcanzar a través de los procesos de enseñanza y aprendizaje. No obstante, puesto que las actitudes tienen una importancia educativa indiscutible, no está de más concienciar al profesorado de la necesidad de incluir experiencias de aprendizaje y criterios de evaluación que fomenten su desarrollo y de trabajar con contenidos que incluyan la adquisición y análisis crítico de normas y valores. Por ejemplo, las actitudes positivas hacia el aprendizaje, el cuidado por los materiales e instalaciones educativas o el respeto y el aprecio de la dignidad humana son actitudes que han de fomentarse siempre que se pueda, en todas las etapas y áreas, pero son más un objetivo o una competencia básica a lograr que un contenido en sí mismo. El fomento de determinados valores y actitudes están implícitos en la cultura de la institución escolar, en su estructura organizativa, en el tipo de relaciones que promueve, en las normas y procedimientos empleados; por ello deben impregnar el proyecto educativo del centro y estar en consonancia con las programaciones y, lo que es más importante, con el desarrollo curricular, es decir, con la puesta en práctica real del modelo educativo propuesto.

En cambio, parece más clara la consideración de los procedimientos como contenidos curriculares. Un procedimiento es un conjunto de acciones ordenadas orientadas a la consecución de una meta. Se refieren, por lo tanto, a la forma de hacer algo, de saber-hacer, lo que cabe también bajo la denominación de destrezas, técnicas o estrategias ya establecidas que se pueden enseñar y aprender. Por ejemplo, métodos de análisis de textos, de recogida de información, de síntesis, técnicas de elaboración de mapas, etc.

\subsection{Los contenidos como elementos del diseño curricular}

El currículum es una elaboración pedagógica que se propone como guía para la acción educativa en la que se plasma una teoría de la educación, de la enseñanza y del aprendizaje. Sea cual sea su concepción y el orden de prioridad que otorgue a cada elemento, es un sistema coherente en el que se articulan objetivos, procesos de aprendizaje, contenidos explícitos y latentes (Vázquez, 1985), procesos de evaluación, y, aunque no suelen tenerse en cuenta, diseños espaciales y modelos organizativos.

El currículum es un instrumento facilitador de la enseñanza y del aprendizaje que permite recrear una parte de la cultura, cuya necesidad viene impuesta por la 

CRITERIOS DE SELECCIÓN DE LOS CONTENIDOS DEL CURRÍCULUM

imposibilidad de aprender todo en el contexto en que se vive. Los mecanismos de educación natural o espontánea ya no son suficientes para asegurar el desarrollo personal adaptado al medio ni el desarrollo social, y puesto que es imposible aprender todo por experiencia directa, tendemos a formalizar los aprendizajes y a crear instituciones y dispositivos que sistematicen las influencias, de manera que se potencien unas y se inhiban otras (Trilla, 1985; Colom, 1987). Uno de los instrumentos en ese proceso es el currículum, en sus muy diversas concepciones, y uno de sus elementos los contenidos.

La educación requiere comunicar contenidos; sin embargo, el papel que se les ha otorgado dentro de las diferentes teorías del currículum ha sido muy diferente (Vázquez, 1999; Colom, 1994; Pérez, 1988; Zabalza, 1991). Dentro de algunos modelos ocupan un lugar central; para otros, por el contrario, sólo son un instrumento al servicio de los objetivos, y en otros, por razones de orden psicológico y sociológico, se tiende a anteponer el desarrollo de los procesos a los contenidos.

En la actualidad, la tendencia es reconocer su importancia, pero resaltando la necesidad de tener en cuenta otros elementos que, formando parte del proceso educativo, influyen poderosamente en los aprendizajes. Los contenidos aparecen estrechamente relacionados con la forma de enseñarlos, con las experiencias de aprendizaje, con los objetivos educativos. En este sentido, la materia de enseñanza, por sí misma, y en función de sus propiedades inherentes, no puede producir el desarrollo completo de la personalidad, ni siquiera de los esquemas cognitivos.

Por lo tanto, los contenidos escolares son un producto cultural específico distinto de aquél del cual proceden (Blanco, 1994, p. 255; Pérez, 1988, p. 73), que requieren una reelaboración pedagógica que no puede identificarse, bajo ningún concepto, con una síntesis o simplificación de aquéllos. Esa reelaboración requiere competencias pedagógicas porque es, en muchos aspectos, una cuestión pedagógica. En cada experiencia de aprendizaje intervienen: el contenido o de lo que trata (datos, conceptos, procedimientos), los procesos cognitivos con los que se trabaja el contenido (memorizar, aplicar, evaluar, etc.) y los sentimientos y actitudes que pone en marcha la forma en que es percibido. Esta es una de las razones por la que la selección de contenidos no es una cuestión exclusiva de los representantes políticos, ni sólo de los expertos en las materias, sino que, en cuanto que se subordinan a fines educativos y se relacionan con los demás elementos del currículum para ser desarrollados en la práctica, adquieren la condición de problema pedagógico. Esto es tanto más cierto cuanto más se avanza en los respectivos niveles de decisión (diseño curricular base, aportaciones de las comunidades autónomas, proyecto de centro y programaciones).

\subsection{Criterios y fuentes de selección de contenidos}

En el proceso de selección de contenidos intervienen varias fuentes que es necesario tener en cuenta para extraer criterios pedagógicos de selección de los 
contenidos. A los efectos de este trabajo las vamos a resumir en tres: la herencia cultural sistematizada en campos de conocimiento, las necesidades y demandas sociopolíticas y el sujeto que aprende con sus características (Wheeler, 1976, pp. 16-27; Pérez, 1988, p. 118). Cada una de ellas plantea unos problemas y, a su vez, unas aportaciones que pasaremos a analizar en los epígrafes siguientes:

a) La herencia cultural está formada por un repertorio evolutivo de experiencias cognitivas, afectivas, instrumentales y morales; depuradas, ordenadas y sistematizadas por las artes, los sistemas de pensamiento, la ciencia, las tecnologías, las tradiciones, etc. Más concretamente, una de las fuentes más importantes de la que se seleccionan los contenidos del currículum es el conjunto de disciplinas que constituyen los conocimientos vigentes. En este terreno, al aumento de conocimientos, a su progresiva especialización y bifurcación en disciplinas, se añade la crisis epistemológica que cuestiona el propio concepto de conocimiento, sus tipos, sus posibilidades y sus límites. Hemos pasado de la creencia en un conocimiento objetivo, estable y con pretensiones de validez universal a un conocimiento consistente en representaciones de la realidad discutibles. El conocimiento parece haberse subjetivado y relativizado. Todo parece cuestionable e interpretable, de modo que el proceso de construcción del conocimiento y su significado se anteponen a su consideración como producto cerrado y acabado. Por ello, muchos profesores se sienten desconcertados al observar cómo se van desvaneciendo los fundamentos teóricos que hasta ahora mismo habían servido para legitimar su práctica.

b) En segundo lugar, podemos citar como fuentes de la selección de los contenidos las necesidades y demandas que se hacen a la escuela, en un momento histórico determinado, desde el ámbito sociopolítico. Los aspectos socio-políticos que han de orientar el currículum y la selección de contenidos también añaden dificultades complejas, difíciles de resolver. La propia pluralidad social genera demandas educativas diversas y hasta contrapuestas.

Puesto que en este trabajo hay un apartado específico para tratar algunas de estas cuestiones, bastará aquí avanzar algunos interrogantes. ¿Es la cultura escolar elitista y, en consecuencia, sus contenidos introducen de facto la desigualdad en el sistema escolar? ¿Es el currículum un mero instrumento de poder ideológico al servicio de la reproducción o es posible introducir contenidos y procesos que fomenten la autonomía, la crítica, la innovación y el cambio? ¿Quién ha participar y de qué manera en el proceso de selección de los contenidos? ¿Es posible romper los intereses corporativistas que hay detrás de las materias a la hora de seleccionar los contenidos? ¿Cómo construir un currículum que no reproduzca los estereotipos de género tradicionales? ¿Cómo dar respuesta a una realidad social multicultural sin caer en el relativismo ni en la asimilación por parte de la cultura más fuerte? 
Junto a estas cuestiones, sin duda, la sociedad actual, en función de sus necesidades y de sus prioridades políticas, económicas, etc. plantea a los individuos una serie de competencias básicas que exigen tener en cuenta ciertos contenidos como los que la propia Logse establece como ejes transversales, así como otros que tienen que ver con el dominio del lenguaje oral y escrito, el uso y la comprensión de los recursos informáticos, el dominio de técnicas para trabajar con la información, para seguir aprendiendo, la comprensión del lenguaje matemático, tener una mínima comprensión científica del mundo, comprender la necesidad de normas de convivencia y saber participar en la toma de decisiones, conocer y comprender los problemas, etc.

c) Por último, a la hora de seleccionar los contenidos habrá que contemplar también las posibilidades de aprendizaje, las necesidades y los intereses de los alumnos, su nivel de desarrollo emocional, relacional, moral, físico, cognitivo, sus experiencias previas, etc.

Los criterios psicológicos son necesarios a la hora de decidir qué enseñar, en qué momento, de qué manera, con qué ritmo, etc. Su importancia, por lo tanto, desborda el marco de lo que es la selección de los contenidos, pero por sus múltiples implicaciones con todos los demás elementos del currículum ya indicados han de ser tenidos igualmente muy en cuenta. Baste, por el momento, con estas consideraciones a las que más adelante dedicamos otro apartado específico.

Como ya hemos indicado, cada una de las fuentes de selección de los contenidos anteriormente mencionadas - la epistemológica, la sociopolítica y la psicológicaplantea interrogantes y problemas que pasaremos a analizar seguidamente. También de cada una de ellas es posible extraer criterios que nos ayuden a establecer prioridades en la determinación de los contenidos para la educación básica. No obstante, creemos que de forma sintética podríamos resumirlos en los siguientes:

a) Criterio de congmencia e idoneidad para con las finalidades educativas y los procesos que se pretende activar en los alumnos. Aunque el profesor tenga su campo de actuación acotado, su poder y responsabilidad son grandes; por eso debe tener claros los procesos y los aspectos de la personalidad que antepone a los demás. ¿Qué les pedirá a los alumnos que hagan: memorizar, analizar, sintetizar, valorar...? ¿Qué tipo de experiencias les propondrá: cognitivas, afectivas, sociales, morales? ¿Se dejará llevar por la rutina y ni siquiera se planteará estas cuestiones? ¿Fijará su atención en la lógica de la materia y se olvidará de la necesidad de provocar experiencias integradas para que el alumno reelabore los contenidos? En función de las respuestas, así serán los contenidos seleccionados y su grado de interrelación, coherencia y potencialidad emocional. Como dice el profesor Vázquez, "ahora sabemos que la significación de lo aprendido, su capacidad estructuradora, interpretativa y de transferencia (su contribución 
al pensar) no depende tan sólo de lo que se contiene en las unidades de información, sino de cómo se utilice el conocimiento por el sujeto que aprende" (1999, p. 32).

b) Criterio de significatividad y reelaboración. Los contenidos deben posibilitar la comprensión de la realidad natural y social, su génesis histórica y su configuración actual, desde un punto de vista teórico y práctico. Pero esa comprensión ha de facilitar la reformulación subjetiva del mundo real y su capacidad para seguir aprendiendo de forma más autónoma, por lo que se ha de partir de lo que ya conoce, de su cultura próxima hasta llegar a comprenderla como un caso concreto de cultura, como una manifestación específica y mudable de una naturaleza humana común (Pérez, 1998; Savater, 1997, p. 161).

c) Criterio de adecuación a los intereses y necesidades de los alumnos. Muchos de los objetivos, de las experiencias y procesos de aprendizaje, permiten optar por una variedad de contenidos. Este criterio establecería la conveniencia de elegir aquéllos que son próximos a la cultura de los alumnos, que por su forma de presentación son pertinentes a su nivel de desarrollo y que enlazan con los intereses inmediatos de ese grupo de alumnos en concreto.

d) Criterio de utilidad y de coherencia con las demandas y necesidades sociales. Al igual que en el criterio anterior, es posible seleccionar entre muestras de contenidos equivalentes aquéllos que, de una forma crítica, conecten mejor con las demandas y necesidades sociales, no sólo con las de la economía, que son instrumentales, sino también con las que nos hacen solidarios y potencian el desarrollo de los derechos humanos: igualdad de sexos, interculturalidad, educación cívica, educación ambiental, educación para la salud, etc.

\section{CRITERIOS EPISTEMOLÓGICOS}

\subsection{Identificación del problema}

Las sociedades modernas, cada vez que han afrontado la reforma del sistema educativo, lo han hecho con el argumento visible de una mayor adecuación a las necesidades de la sociedad; buena parte de esas necesidades se han definido siempre en términos de correspondencia con la evolución de los requerimientos de los modos y formas de producción y de organización social. Pero además de este argumento, el más visible y recurrente, o mejor dicho, detrás de este argumento, pueden detectarse históricamente algunas constantes que vendrian actuando de principios invariantes.

De un lado, el propio hecho del desarrollo del conocimiento, pero sobre todo la creencia en una expansión cuantitativa, arborescente y disciplinar del conocimiento que ocuparía, progresiva y ordenadamente, todos los intersticios de la rea- 
lidad, primero natural y después social, y que obligaría por sí misma, pero sobre todo por las características atribuidas a ese conocimiento, a revisar periódicamente la cantidad y el tipo de contenidos a concretar en el currículum del sistema; este principio estaba llamado a convertirse en problemático cuando se diese al menos uno de los siguientes supuestos: el crecimiento exponencial de los conocimientos y/o el cuestionamiento de la visión ahí implícita del propio conocimiento y la propia realidad. En ambos escenarios, aunque por distintas razones, la dialéctica que en toda reforma curricular se plantea entre lo viejo y lo nuevo, entre la cultura que un pueblo interpreta como ya consolidada y el conocimiento que entiende imprescindible para su desarrollo, se vuelve problemática, sobre todo cuando no es muy del uso la aplicación del primer principio propuesto por la Comisión para el estudio de los contenidos de la enseñanza en la escuela francesa: "todo elemento añadido deberá compensarse con una supresión concomitante" (Bourdieu y Gros, 1990). Con todo, y pese a todas las dificultades que conlleva esta primera invariante a la hora de determinar los contenidos de la enseñanza, parecen deducirse dos consideraciones que no por obvias deben menospreciarse: primera, que sin contenidos no hay enseñanza ni educación y, segunda, que en sociedades complejas en intereses, cultura y modos de producción, difusión y utilización del conocimiento los contenidos curriculares quedan sujetos a revisiones periódicas, incluido el propio sentido y uso del conocimiento por los sujetos. La primera consideración no siempre ha sido tenida en cuenta por parte de la investigación educativa (Gimeno, 1992,1998) y con base en la segunda se han hecho reformas que no han ido más allá de la mera adición de contenidos a los ya existentes, por ignorar los modos de vida y usos del conocimiento en los sujetos.

Precisamente, el segundo principio invariante que encontramos en las reformas curriculares de la modernidad hace referencia al sujeto; todas las reformas educativas modernas tienen y construyen, junto a la cultura acumulada y el conocimiento reciente, "Su" sujeto escolar. Una vez más conviene que nos detengamos a reflexionar en lo que parece una obviedad. Plantea el profesor Gimeno en uno de sus últimos trabajos que "el reto incumplido de la pedagogía moderna aún hoy" viene dado por la falta de un nexo significativo entre cultura y sujeto, entre el concepto objetivo de cultura como realidad externa al sujeto y el sentido subjetivo de cultura como apropiación de ésta por el sujeto, nexo que la modernidad introdujo recuperando la mejor interpretación clásica y humanista de la formación humana (Gimeno, 1998, pp. 196 y ss.). Más aún, llega a dudar el autor de que los sistemas escolares actuales sean capaces de canalizar las sucesivas ampliaciones y transformaciones que la postmodernidad ha hecho de la cultura y del sujeto de la educación; dicho en términos que Colom y Mélich recogen del pensamiento postmoderno, el problema está en que la escuela es moderna y los alumnos son posmodernos (Colom y Mélich, 1994).

Es cierto que el sujeto está presente en los planteamientos de la tradición moderna y que las sucesivas reformas curriculares de la modernidad han ido balanceándose entre los dos polos del binomio, el sujeto y la cultura objetiva; pero acaso 
el problema radique en la forma originaria como la modernidad estableció, primero, la frontera entre las dos orillas, el sujeto y el mundo exterior, así como la relación epistémica y de acción que les comunicaba. Hecha así la distinción y separación en términos cartesianos y tendido el puente instrumental de la Razón Objetiva para transitar, no sólo quedó configurado el acto educativo de la modernidad como el tránsito que debe realizar el sujeto a través del conocimiento del exterior (Pineau, 1999), sino que también quedó conformada una visión de la educación y del conocimiento como prótesis que se implanta al sujeto para su desenvolvimiento en la realidad. No extrañará, pues, la comodidad con la que nos movemos cuando, en el caso de la selección de los contenidos curriculares, adoptamos el punto de vista disciplinar de una realidad suficientemente fragmentada como para ser conocida y dominada y las dificultades que encontramos cuando lo que se pretende es sumergir al sujeto en un proceso de subjetivación y experienciación del conocimiento de una realidad convivida y construida por el propio sujeto y, por ende, por todos los sujetos, realidad que, si se es consecuente con el planteamiento, no puede ser otra para empezar que la más próxima a cada sujeto. En este segundo caso, la solución quizás no venga de la búsqueda de nexos entre lo uno y lo otro, búsqueda que termina escorándose inevitablemente hacia lo uno o hacia lo otro, sino de la negación de la interpretación que hizo la tradición moderna de esos dos elementos como instancias separadas, autónomas e independientes; no otra es la pretensión de las múltiples variantes que se recogen bajo el manto de la corriente constructivista e interpretativa.

\subsection{Formulación del problema}

Diríamos que desde hace unas décadas y en el futuro próxımo el problema, planteado con rotundidad, se presenta en los siguientes términos: ¿cabe todavía hablar de criterios epistemológicos para seleccionar los contenidos del currículum, cuando por una u otra vía, ya sea desde el sujeto o desde el mismo conocimiento, se termina cuestionando la validez de la cultura como cuerpo de conocimientos y estructuras cognitivas objetivas, estables y válidas universalmente? No, desde luego, si seguimos pensando en términos de epistemología clásica que, por enfatizar determinadas características metodológicas, terminó expulsando al sujeto del propio proceso de conocimiento; pero ello no significa ausencia total de una visión epistemológica en la configuración del currículum, entre otras razones porque el sentido procesual de este concepto, incluyendo aquí su dimensión visible, oculta y ausente, conlleva inevitablemente una forma de ver, interpretar y usar el conocimiento y la realidad. En última instancia, lo que aprende el sujeto, con unos u otros contenidos, no sólo son conceptos, procedimientos y valores, sino fundamentalmente formas de conocer y enfocar la realidad que terminan por condicionar ese conglomerado conceptual, procedimental y actitudinal; de ahí que no resulte indiferente en la selec- 

CRITERIOS DE SELECCIÓN DE LOS CONTENIDOS DEL CURRÍCULUM

ción de los contenidos la visión epistemológica utilizada, como tampoco lo son los contenidos a utilizar una vez decidida la opción epistemológica.

Dicho esto y formulado así el problema, conviene avanzar ya que en estos momentos la visión epistemológica más adecuada para llevar a cabo la selección de contenidos del currículum de la enseñanza obligatoria, y, por tanto, también los posibles criterios susceptibles de ser extraídos no deben ignorar la reconceptualización que han conocido el sujeto y la cultura en los últimos tiempos, comenzando por cortar distancias que siempre requieren después de algún puente. Pero antes de entrar en el desarrollo de esta temática, nos interesa dejar constancia de algunas investigaciones realizadas sobre lo que podríamos llamar "comportamientos en la práctica curricular" de selección de contenidos, por si nos pueden proporcionar algo de luz sobre "el valor en uso" de los criterios epistemológicos para esa tarea.

\subsection{Uso y valor de los criterios epistemológicos}

Sabemos que son varias las instancias que intervienen en la selección de los contenidos del currículum y múltiples los criterios generales que pueden mover la actuación de esas instancias (Ibáñez-Martín, 1987); en nuestro caso, la propia administración educativa estableció en su momento distintos niveles de decisión y concreción curricular. Por otra parte, desde el primer momento se señalaron, para dar respuesta a algunas preguntas fundamentales del currículum, entre otras la relativa a "qué enseñar", cuatro fuentes de naturaleza y origen diferentes a tener en cuenta en todas las fases de elaboración y realización del currículum: una de tipo sociológico, otra psicológica, una tercera de carácter pedagógico y la fuente epistemológica. Nos interesa ahora detenernos en ésta última: ¿cómo se concreta en los distintos momentos, fases e instancias por las que atraviesa el proceso de selección de contenidos?, ¿qué valor se le atribuye, qué uso se hace de este criterio en la dinámica práctica de la configuración curricular?, en fin, ¿qué interpretación de epistemología subyace?. Contamos con varias fuentes de reflexión.

a) Un grupo de profesoras de la Universidad de Salamanca ha llevado a cabo una investigación para conocer los criterios utilizados por los profesores a la hora de seleccionar, secuenciar y organizar los contenidos (Clemente Linuesa, Domínguez Gutiérrez y Ramírez Orellana, 1999); la investigación se ha desarrollado en centros públicos y privados de educación infantil y primaria de Castilla y León. De los seis criterios utilizados por los profesores al realizar esta tarea, el criterio epistemológico "ocupa una posición poco importante para los profesores, concretamente es el penúltimo entre los seis considerados" $\mathrm{y}$, lo que puede ser más significativo, hay un $60 \%$ de profesores que no contestan a los ítems referidos a este criterio; el más importante para el conjunto de los profesores es el criterio psicológico. Aunque estos resultados no nos sorprenden, pues ya sabemos que este criterio presenta menor peso específico a medida que se desciende en el nivel 
educativo, no sería superfluo formular algunas preguntas al respecto, sobre todo si, como hemos dicho anteriormente, en ningún caso es irrelevante este criterio para la selección de contenidos. ¿Qué significado tiene ese alto porcentaje de profesores que no responden a estas cuestiones?. Lo que no puede ignorarse es la presencia de concepciones epistemológicas en el profesor (Gimeno, 1988).

b) Una línea de interés indudable para la reflexión sobre la selección de los contenidos del currículum, y que afecta directamente al debate sobre la existencia y utilidad de criterios epistemológicos para este cometido, nos ha llegado recientemente de la mano de los estudios comparados sobre el contenido de los curricula escolares nacionales (Benavot y otros, 1991; Meyer y otros, 1992). El estudio longitudinal y transnacional realizado por el primer grupo para el período comprendido entre los años 1920 y 1986 - para las dos últimas décadas los autores utilizaron datos de aproximadamente 125 países - resalta la existencia de "curricula escolares sorprendentemente afines" en la escuela primaria tanto en lo que respecta al núcleo de materias básicas que configuran los curricula oficiales como en la asignación de períodos lectivos a esas materias, más allá de "diferencias nacionales en la estructura política, económica y social". Cuestionando en parte algunos supuestos de las teorías funcionalistas e historicistas, parece deducirse que "un currículum mundial cada vez más semejante ocupa la mayor parte del tiempo lectivo. Estos hallazgos señalan que durante gran parte del siglo XX ha estado operando un currículum mundial estándar para la educación primaria". Acogiendo también nosotros en parte con sorpresa estos resultados -no siempre se enseñaron las mismas materias ni en cada materia se enseñaron los mismos contenidos; el mismo estudio recoge un importante epígrafe sobre limitaciones de los datos: ausencia de información sobre los contenidos específicos de las materias enseñadas... categorías generales afines pueden enmascarar una variabilidad nacional de los contenidos proyectados... o a través del tiempo... además de procesos organizativos que pueden aumentar las diferencias-, y recordando que una cosa es el currículum oficial y otra el currículum real, no conviene ignorar que "las definiciones oficiales de lo que deben estudiar los alumnos son interesantes en sí mismas... especificando los contenidos de la formación... y establecen los límites sobre ciertos conjuntos de conocimientos considerados apropiados".

Cuando los autores tratan de explicar los procesos por los que se logra esta estandarización curricular, acuden a tres tipos de fuerzas difíciles de distinguir empíricamente: un proceso de clonización del estado-nación que incluye determinados aspectos del racionalismo técnico moderno, la lógica de un modelo de educación de masas que enfatiza y conforma progresivamente un contenido cultural común y la influencia de determinadas organizaciones internacionales. Con todo, no logran desembarazarse de 
una duda radical que les asalta y terminan preguntándose si «existe una lógica cultural sustantiva o se trata simplemente de la aplicación de pautas accidentales o sistemáticas de la dominación internacional". La profesora Nieves Blanco ha sintetizado perfectamente las posibles hipótesis que pueden plantearse: “La primera encontraría apoyos de tipo epistemológico: esos núcleos de contenido representan formas universales de conocer de los seres humanos y, por tanto, es lógico que no existan diferencias. Una segunda hipótesis plantearía la existencia de una lógica cultural sustantiva: existen invariantes universales más allá de las diferencias culturales o temporales... Una tercera hipótesis plantearía esa homogeneización como la evidencia de la imposición de las pautas culturales occidentales... Ni la investigación ni la teorización proporcionan, de momento, respuestas para despejar estas hipótesis" (Blanco, 1994).

c) La tercera línea de reflexión nos viene del análisis del documento presentado por la Comisión para el estudio de los contenidos de la enseñanza en la escuela francesa, ya aludido anteriormente. En la declaración de intenciones formulada al inicio de su trabajo manifiestan su deseo de «ajustarse en todo momento a la disciplina propiamente intelectual emanada de la lógica intrínseca de los diversos ámbitos del saber"; no obstante, de la lectura atenta de los siete principios propuestos para renovar progresivamente los contenidos de la enseñanza primaria se extraen algunas conclusiones de interés para nosotros. En primer lugar, encontramos ya desde el primer principio la presencia combinada de criterios propiamente epistemológicos con otros de tipo social, psicológico y pedagógico, adquiriendo éstos últimos un peso notable en el conjunto de las propuestas formuladas; no se detecta aquí, sin embargo, un reduccionismo metodológico muy al uso cuando se afronta la tarea de revisar los conocimientos transmitidos en la escuela. Más bien sucede al contrario, desde el inicio y a lo largo de su propuesta se previene de la tentación de primar contenidos y enseñanzas que resultan "más agradables" sobre aquéllos otros que se entienden más o menos indispensables, exigibles. En segundo lugar, ¿cómo resuelven o aplican ese criterio de necesidad o exigibilidad?. Sin dejar de reconocer que no resulta fácil discernir siempre y en todos los dominios lo caduco de lo válido y depositando "la solución, si así puede denominarse, ... a una reflexión permanente sobre la relación que puede y debe establecerse entre la conservación necesaria del pasado y la adaptación no menos imprescindible al porvenir", parece claro que el criterio de decisión viene dado por "los modos de pensamiento dotados de validez y aplicabilidad generales" -se señalan tres modos de pensamiento fundamentales: el pensamiento deductivo, el pensamiento experimental, el pensamiento histórico-; los miembros de la Comisión entienden que las diversas áreas del saber pueden contribuir a la transmisión de los distintos modos de pensamiento sin perjuicio de reconocer que hay contenidos y áreas especialmente útiles, por su tra- 
CRITERIOS DE SELECCIÓN DE LOS CONTENIDOS DEL CURRÍCULUM

dición y naturaleza intrínseca, para asegurar el aprendizaje de uno u otro de esos modos de pensar. Es lo que podríamos llamar el carácter multimodal de los contenidos de una disciplina.

A estos modos de pensamiento fundamentales añaden los autores, en una línea de ampliación del sentido tradicional de contenido, lo que denominan técnicas o métodos racionales de trabajo y estilo crítico, reflexivo y valorativo que siempre debe acompañar a unos y a otros. El resto de consideraciones o principios son en realidad una derivación del principio anterior, combinado en ocasiones con otros criterios de corte psicológico, pedagógico o sociológico, por ejemplo, la progresividad, coherencia e interdependencia recíproca de los contenidos - conexión vertical y horizontal-; la diversificación del estatuto de las distintas enseñanzas -obligatorias, opcionales y facultativas-, que los autores conectan con la diversificación de formas pedagógicas, o la búsqueda del equilibrio e integración entre los distintos contenidos y modos de pensamiento propios de las ciencias de la naturaleza con los de las ciencias del hombre, conciliando el universalismo y el relativismo de unos y otros modos de pensamiento, atentos siempre a las raíces históricas de toda obra cultural y respetando la diversidad en el tiempo y en el espacio de los modos de vida y las tradiciones culturales. En definitiva, toda una matriz de criterios que se sustentan sobre la aceptación de la existencia de lo que denominan modos de pensamiento fundamentales y el reconocimiento de la identidad y especificidad de esos modos de pensamiento en función de grupos de contenidos más idóneos, sin perjuicio de aceptar a modo de una redefinición de la división disciplinar de los contenidos de la enseñanza en función del propio desarrollo de las ciencias.

Buena parte de las posiciones y comportamientos adoptados en las distintas líneas de reflexión presentadas nos retrotraen a perspectivas y orientaciones ya conocidas. En efecto, la insistencia en los modos de pensamiento y su adopción como punto de partida para la revisión de los contenidos, el comportamiento homogéneo de los núcleos básicos del currículum a nivel mundial, al menos en alguna de sus hipótesis explicativas, e incluso la actitud de desimplicación que los profesores mostraban respecto de los criterios epistemológicos, que también puede ser interpretada como la aceptación en la práctica de un supuesto no cuestionado, recuerdan los planteamientos de P. Hirst y Ph. Phenix sobre las formas y estructuras de conocimiento universales y estables y las esferas disciplinares de significado como formas diferenciadas que adopta el conocimiento. Parece como si los trabajos de M. F. Young, B. Bernstein, M. Apple, Th. Popkewitz... en educación y los de T. Kunh y S. Toulmin, P. Berger, Th. Luckmann... o Foucault en cuanto a formas de producción y distribución del conocimiento no hubiesen surtido los efectos esperados. O, lo que también es verosímil, que en esta confrontación que combina perspectivas en ocasiones diferentes, en unos casos epistemológicas y en otros culturales, se hubiese optado por actuar en la práctica en función de una doble estrategia: 
reconocer el carácter social del conocimiento y de toda producción y selección cultural sin renunciar a la búsqueda de un mapa cultural representativo de la dinámica compleja y plural de la sociedad (Skilbeck, 1981; Lawton, 1989; Sarramona, 1989) y, lo que es más importante, a la búsqueda de unas herramientas, enfoques, formas de funcionamiento..., que en ocasiones actúan de criterios de selección de contenidos y en otras de contenidos, que permitan al sujeto no sólo la reproducción del orden cultural sino también la reconstrucción reflexiva y crítica -éste sería el valor añadido de nuestro tiempo a la pregunta permanente por cuál sea el conocimiento más valioso (Vázquez, 1993b)-, comenzando por y terminando en la reconstrucción relativamente autónoma de la cultura experiencial del individuo (Pérez Gómez, 1998).

\subsection{Una epistemología transaccional, relacional, que no relativista}

Conviene aclarar de entrada varias cuestiones. Primero, que no buscamos planteamientos que concilien posiciones; aunque pensamos que esa tarea sería factible, no es nuestro objetivo en este momento. Segundo, que renunciamos, por supuesto, a la pretensión ilusoria de interpretar el criterio epistemológico como el único y/o el más importante a utilizar en la configuración de los contenidos del currículum; de hecho, en la propuesta que hacemos a continuación se deslizarán inevitablemente otros criterios. Y tercero, que seguimos pensando que en última instancia la mayor potencialidad de un currículum propuesto a un alumno reside en la propia visión e interpretación que conlleva de la realidad y del conocimiento de esa realidad, con lo que ello tiene de configuración de comportamientos y acciones sobre esa realidad. En este sentido, si la concepción epistemológica predominante en la escuela moderna resulta hoy inservible, o al menos claramente insuficiente, habrá que pensar en otra, pero no podemos abordar la tarea de seleccionar y organizar los contenidos de un currículum sin tener a la vista ninguna, porque al final una u otra se harán presentes.

Una relectura atenta de la trayectoria que venimos recorriendo en nuestra reflexión identificaría fácilmente los conceptos de cultura, conocimiento, realidad y sujeto como los puntos de apoyo sobre los que descansa la configuración del currículum; ahora bien, de la manera como entendamos cada uno de esos conceptos así como de la forma de conexión que se establezca entre ellos dependerá que terminemos en una u otra visión epistemológica. No es una dificultad menor decidir por cuál de esos conceptos debe empezar nuestro análisis; como suele ocurrir cuando nos movemos en la complejidad, al final parece como si hubiera sido indiferente haber iniciado la reflexión por uno u otro lugar, pero al principio no lo es. En el contexto de este trabajo nos corresponde hacerlo por la interpretación del conocimiento, aunque en verdad sea el binomio sujeto-realidad el referente nuclear de todo el entramado (creemos más acertado hablar y pensar en términos de esa unidad que separarla inicialmente en dos instancias para luego buscar enlaces de conexión entre sus procesos y productos. Gimeno, 1998; Pérez Gómez, 1998). 
La segunda mitad del siglo XX ha conocido transformaciones importantes no sólo en los tipos de conocimiento, en los modos de producción y difusión del conocimiento sino en la misma interpretación de lo que sea el conocimiento. De poner el énfasis en el conocimiento en cuanto producto o resultado de un complejo operativo que, por ajustarse a unas características metodológicas concretas, pasaba a convertirse en una segunda realidad tan objetiva, estable, homogénea y verdadera como la misma realidad en él representada, se ha pasado a una visión del conocimiento no sólo como construcción de interpretaciones y significados susceptibles de ser diferentes sino sobre todo como construcción de la propia realidad al mismo tiempo que se construye el sujeto en su identidad y autonomía; de instrumento de organización y dominio de lo otro y del otro, de la realidad natural y social, en base a la categorización ontológica de esos productos y vía razón universal, se ha pasado a una interpretación como herramienta de subjetivación, de configuración y desarrollo del sujeto, proceso en el que lo otro y el otro, la diferencia, aparecen como instancias imprescindibles de una realidad interaccional, compleja, dinámica, continua. Después de las aportaciones de Kuhn, Toulmin, Berger, Luckmann... Bruner, Vigotsky, Wertsch, Cole... Maturana, Varela... Morin, Prigogine... Gergen, Schnitman, Watzlawick..., sin pretensión ninguna de exhaustividad en corrientes y autores, podríamos decir, parafraseando a A. Touraine, que no hay ninguna discontinuidad entre sujeto y realidad natural y social.

Claro que las tentaciones son muchas, todas ellas emergentes de la polarización que puede producirse en una u otra dirección: en la autonomía e identidad o en la interdependencia y diversidad; en la apertura y coderiva de las culturas o en la clausura de los sujetos y de los pueblos; en una interpretación restringida o amplia de la cultura; en una interpretación de la cultura como patrimonio objetivo de los pueblos a conservar generacionalmente o necesitada y susceptible de reinterpretación para su utilización por los sujetos... y, por último, en el fundamentalismo más exacerbado al tomar la realidad más próxima en el espacio y en tiempo, lo que por otra parte es necesario y congruente con el enfoque global, como único contexto de configuración del sujeto o en el relativismo total, cultural y epistemológico. No podemos detenernos en estos momentos a tratar toda esta problemática y el afán de presentarlo como dilemas. Digamos simplemente que en esta perspectiva no es cierto que todo valga y de igual manera; sería suficiente con respetar las reglas internas a la propia perspectiva, entre las que no es menor el principio-criterio de la necesidad de reconocer y contar con la existencia del otro y de lo otro, en toda su diversidad, si queremos lograr nuestra propia identidad.

Nos interesa más responder la siguiente pregunta, porque nos obliga a una mayor concreción: ¿tiene potencialidad suficiente esta concepción epistemológica para ayudarnos a determinar y configurar los contenidos de un currículum?. No lo dudamos; más aún, encontramos que las vías de configuración y selección de contenidos desde esta perspectiva podrían ser varias. No creemos que fuera incompatible la adopción de los modos de pensamiento, tipos de conocimiento, formas de acción, comunicación... como criterios susceptibles de identificar contenidos idó- 
neos para el currículum, pero probablemente esta opción rezumaría intelectualismo, academicismo, disciplinariedad. Nos acogemos a otra opción inherente al propio planteamiento y que resumimos en los términos siguientes:

a) Esta perspectiva tiene potencialidad suficiente para identificar núcleos de contenido del currículum; basta con hacer visibles las vertientes de relación del sujeto con el otro y con lo otro, enfatizando las formas, modos y lenguajes de relación y comunicación así como la pluridimensionalidad de la identidad del sujeto; no son núcleos y criterios arbitrarios sino que responden a exigencias de la propia perspectiva.

b) Esta perspectiva tiene la potencialidad suficiente y la versatilidad necesaria para identificar y seleccionar contenidos en cada núcleo y para organizar y estructurar los núcleos y contenidos de forma diversa, en función de los requerimientos exigidos por otros criterios; en este sentido la propia perspectiva impone algunas restriciones: no serán contenidos adecuados los que, por sí mismos o por su presentación, contradigan o recorten las vertientes relacionales, formas comunicacionales y dimensiones de la identidad del sujeto. Esta perspectiva no impone límites cuantitativos, al contrario, aconsejaría versiones plurales de un mismo contenido, pero la escuela sí los tiene; en este aspecto, la perspectiva introduce un criterio de calidad del contenido en función de su potencialidad, valga la reiteración, para contemplar el mayor número de vertientes y dimensiones referidas anteriormente.

c) Esta perspectiva aclara el debate entre lo común y lo diverso y lo hace no precisamente en términos de capacidades del sujeto o formas pedagógicas; diríamos que aquí la diversidad no sólo es un valor sino una exigencia impuesta por la propia naturaleza y dinámica del sujeto, que comienza a configurar su identidad en base a un espacio y tiempo cultural concreto.

d) Esta perspectiva no es indiferente a los valores ni cualquier valor es compatible con ella. El enfoque que hemos adoptado conlleva y exige la aceptación de algunos valores básicos, entre los que hemos señalado alguno de manera reiterada pero consciente, y desde luego no requiere de artificios pedagógicos transversales.

Parece un atrevimiento que lo que empezó como epistemología termine como antropología (Escámez, 1987), después de habernos zambullido por la ontología; no podía ser de otra manera. Si aislamos los criterios epistemológicos, procurando evitar cualquier otra "contaminación", probablemente no haya salida; algo parecido ocurrió en los inicios de la modernidad. Por último, no se nos oculta que quedan por hacer muchas otras consideraciones, pero no queremos que se nos olvide una: esta perspectiva no reniega ni declara inservible el legado cultural, ya sea en sus formas de conocimiento, de acción o de comunicación, pero está más interesada en que el sujeto lo pueda recrear al tiempo que pueda seguir creando cultura. La abundancia de conocimientos, lenguajes... información (contenidos/lenguaje) característica de nuestro tiempo no debe ser una dificultad epistemológica añadida, 
si superamos el riesgo de que cada reforma curricular genere su propio y característico sujeto escolar y le dotamos de herramientas de comprensión, integración y valoración crítica de la información.

\section{CRITERIOS SOCIOPOLÍTICOS}

Cada día muchas personas de profesiones diversas, profesores, editores, escritores, políticos, administradores, etc., se encargan de reelaborar el mundo cultural, de crear nuevas figuras, contrafiguras, modos de saber, que luego la escuela reconstruirá con su propia práctica. Como observó Bernstein, "la forma en que una sociedad selecciona, clasifica, distribuye, transmite y valora los saberes destinados a la enseñanza, refleja la distribución del poder existente en su seno y el modo en que se encuentra garantizado en ella el control social de los componentes individuales" (Bernstein, 1971). Ciertamente esto es así, el currículum se encuentra mediatizado por procesos políticos y sociales complejos y a los que desde hace un tiempo venimos prestando atención con resultados desiguales.

En un trabajo reciente, el profesor Puelles Benitez señalaba la falta de atención que la comunidad científica había prestado a los principios políticos y sociales que informan el currículum. A su juicio esa falta de atención era mayor entre los politólogos que apenas se habían ocupado del tema, en tanto que los sociólogos habían intentado diversos desarrollos, pero en ambos casos los resultados eran poco alentadores; ni unos, ni otros, lograban alcanzar un mínimo desarrollo teórico consistente.

La preocupación por el estudio de la interacción sociedad-currículum puede encontrarse en los orígenes de la Sociología de la Educación. Durkheim la abordó en La Evolución Pedagógica en Francia, con un penetrante análisis de las complejas relaciones entre la estructura social y los procesos de transmisión educativa, pero su esfuerzo no se vio continuado hasta casi sesenta años después. Lo mismo se puede decir del análisis que Dewey realiza de la interacción política-comunidadescuela, en el intento de fundar su filosofía de la educación. Lo cierto es que sólo a partir de mediados de los sesenta, principios de los setenta, y en el ámbito anglosajón, estas cuestiones cobraron cierta relevancia. La Sociología del Currículum -que tuvo desarrollos de interés en Inglaterra, Estados Unidos y, en menor medida, Canadá y Australia-, en algunos momentos pareció que podría llegar a consolidarse como el campo más fructífero para el análisis social de la educación, pero hoy, casi treinta años después, esa posibilidad parece haberse esfumado. En países como España, Francia y Alemania, esta influencia se hizo sentir en la década de los 80 , pero en esta década el interés ha decaído. En España los trabajos del malogrado prof. Lerena son, quizá, los más relevantes e interesantes, y dieron un cierto impulso a este tipo de investigaciones, pero en la actualidad, sin dejar de publicarse algunas cosas, han desaparecido de las revistas especializadas. Donde sí parece tener cierto vigor y vigencia es en nuestro campo, muy especialmente en el 
de la didáctica, pero muchas de las aportaciones siguen sin superar los principales obstáculos y problemas señalados por Young (1990): debilidad metodológica, falta de un marco teórico consistente, circularidad de los planteamientos. En definitiva, no se hace más que reiterar algunas ideas bien conocidas, pero no por ello más acertadas o verdaderas.

¿Qué hechos pueden señalarse como causas de ese abandono y de esa falta de resultados y continuidad en la investigación?. Sin pretensiones de exhautividad y dentro de los límites de este apartado en este artículo, nos gustaría señalar algunos, aunque sean en sus rasgos más generales. Puelles Benítez apunta como causa de esa falta de resultados, el interés por investigar cuestiones relativas a las "policy" (políticas concretas que se implementan) en detrimento del análisis de las "politics" (análisis del conflicto, del debate de las ideas políticas), que ha llevado a preocuparse más de aquellas prescripciones y normas en torno al qué, cómo, cuándo y para qué (esto último en menor medida) enseñar, emanadas de los distintos poderes políticos, de las distintas administraciones. Ciertamente así ha ocurrido y seguramente es una causa, pero deben buscarse causas internas a los propios procesos de análisis realizados. En esta línea señalar:

a) El intento de muchas investigaciones por mostrar la exclusividad o elitismo social del conocimiento académico les llevó a reemplazarlo por contenidos de la conciencia popular o del sentido común, sin realizar un análisis crítico de la propuesta que se estaba haciendo. Estos planteamientos tuvieron como resultado algunas consecuencias como las que se han visto en el caso inglés y empiezan a apuntarse, con razón o sin ella, en nuestro sistema educativo.

b) La fuerte tendencia a realizar puras descripciones del currículum, sin adoptar una perspectiva analítica e interpretativa, que tuvo como consecuencia ver el currículum como un puro instrumento de poder ideológico sin percibir otras virtualidades, y vaciando a la escuela de contenido y especificidad. Desde esa postura se hace difícil explicar por qué los alumnos aprendían, mal que bien, a comprender mejor el mundo, a instalarse en él, a resolver problemas concretos, a orientarse.

c) Como consecuencia de esa devaluación del currículum, reduciéndolo a puro instrumento ideológico, carecen de criterios para desarrollar y valorar alternativas curriculares.

Otro factor, sin duda influyente, es la propia complejidad que el análisis exige, y la multiplicidad de puntos de vista y perspectivas que se pueden adoptar. Si, como indica el prof. Sarramona $(1999$, p. 8), la fundamentación pedagógica del currículum implica dar respuesta al qué, cómo, cuándo, dónde enseñar, qué y cómo evaluar, qué materiales elaborar, todas y cada una de esas preguntas pueden presentarse como preguntas de contenido sociopolítico, es decir, pueden ser vistas y analizadas como condicionadas por la perspectiva sociopolítica. Cuando nos preguntamos quién debe definir el currículum, inmediatamente se ponen en juego 
diversas fuerzas sociales comprometidas en ello. Para unos el Estado tiene unas responsabilidades innegables, para otros deberían ser las comunidades más próximas las encargadas de perfilarlo. Otros abogan por organizaciones políticas de carácter mundial, e incluso se defiende que sólo desde la visión globalizadora o ambientalista esa construcción es posible. Los hay que estiman que debe ser la propia comunidad escolar, o bien, los técnicos en educación, los profesores. Y, cómo no, existen muy diversas posiciones de síntesis y sincréticas.

Podría ejemplificarse con cualquier otra de las dimensiones señaladas, y las preguntas y perspectivas se multiplicarían. ¿Cómo abordar, pues, esta complejidad?. La perspectiva que adoptamos aquí puede ser discutida y considerada insuficiente, pero nos ha parecido operativa dentro del espacio que tenemos asignado. Nuestra perspectiva adopta como criterios de ordenación dos ejes fundamentales, uno externo y de carácter histórico, el otro interno y de carácter más claramente epistemológico.

El primero hace referencia a lo que a nuestro juicio ha constituido el núcleo de la discusión de la política educativa los últimos ciento cincuenta años, y que después de la II Guerra Mundial se ha definido como la polémica en torno a la igualdad. La discusión acerca de cómo construir una igualdad educativa y el diseño de políticas encaminadas a conseguirlo nutre todas las discusiones sobre el currículum y, muy especialmente, sobre su contenido y sus implicaciones sociales. El currículum como instrumento de exclusión de clases, como forma de dominación, como correspondencia de relaciones sociales de producción, etc. También dio pie a la defensa de propuestas alternativas, currículum como contrasocialización, currículum político, etc.

El segundo eje hace referencia al debate en torno a la segregación e integración de disciplinas, que traduce a términos escolares la vieja cuestión de la posibilidad de unificación de la ciencia y la cultura, y que arrastra tras de sí toda una suerte de cuestiones de fuerte calado epistemológico. Cuestiones éstas que se proyectan en todo el ámbito de las ciencias sociales y particularmente en el de las ciencias de la educación. Esta perspectiva lleva a plantear toda la discusión acerca de las tesis del aprendizaje implícitas en educación, qué límites tiene el conocimiento, la relación biología-educación, cultura-culturas, etc. Esta polémica tuvo su origen en Inglaterra a raíz de las propuestas de P. Hirst, que, -aún defendiendo como fundamental para una Educación Liberal el acceso de todos las personas a los saberes más complejos, según su capacidad y esfuerzo- afirmaba que cada saber, independientemente de las relaciones funcionales que guarde, obedece a su propia lógica interna y es independiente de los otros. El debate fue tan intenso que llamó la atención de otros investigadores no vinculados al campo de la filosofía y la pedagogía, en concreto, los sociólogos.

La investigación sociológica se centró en averiguar qué ocurría en el interior de la escuela, por qué se producían resistencias al cambio, por qué no se aceptaba, en muchos casos, la integración curricular de la disciplinas, etc. Para poder explicar lo que ocurría tuvieron que suponer que las materias escolares no actuaban sólo como disciplinas, como sistemas intelectuales, sino que también constituían 
auténticos sistemas sociales, comunidades que fijan fronteras, exigen fidelidad y dan identidad a sus miembros. De este modo la discusión que comenzó en el campo de la Filosofía se transformó en un debate sociopolítico que desembocó en el análisis sociológico de la circulación, construcción y desarrollo de los saberes escolares.

Ambos ejes no se presentan como líneas separadas que se prolongan en el espacio sin rozarse siquiera, sino que entre ambas se producen múltiples cruzamientos y relaciones complejas. No podemos ocuparnos aquí de los desarrollos que siguieron, pero sí queremos resaltar que la interrelación de ambos ejes encuadra los principales criterios de selección de contenidos curriculares. En realidad, las líneas en sí funcionan como criterios de selección: rigor y coberencia científicopedagógica y profundización en la igualdad, a los que podría añadirse un tercero, que está dado implícitamente, construcción de una identidad más libre. Precisamente, desde las perspectivas indicadas, nos ocuparemos de tratar a continuación dos de las problemáticas que actualmente más se están debatiendo y que están en el centro de las cuestiones sociopolíticas del currículum: igualdad y género, y cultura, etnia y educación.

\subsection{Igualdad y educación: el caso del género}

Durante mucho tiempo la lucha por la igualdad social fue entendida como una lucha por la igualdad entre grupos sociales. Se trataba de que las clases más desfavorecidas alcanzaran mejores niveles económicos, sanitarios, educativos o culturales, pero en ningún momento pareció necesario establecer ningún tipo de diferencia en estos proyectos que contemplase la existencia diferencial de hombres y mujeres. Se pensó que, alcanzados determinados niveles sociales, éstos incidirían por igual en los dos géneros. Sólo después de la II Guerra Mundial, y como fruto de la reflexión acerca del trabajo que las mujeres desempeñaron en la guerra y en la reconstrucción de la economía después del conflicto, se comenzó a cobrar conciencia de que no era así.

Desde ese momento comienza una larga marcha de cambio y transformación de la escuela y del currículum escolar. En España ese camino se comenzó a andar más tarde. La Ley General de Educación de 1970 concretará en principio legal la existencia de una escuela mixta, que no sin dificultades sentará en los mismos bancos a niñas y niños. Salvo la supresión de alguna materia específica, en esta primera etapa, poco más se hizo. Mediada la década de los 80 se empieza a plantear otro tipo de escuela. Una escuela que tenga por objetivo la modificación radical de las relaciones interpersonales. Para ello sitúa el problema en unas nuevas coordenadas: se trata de cambiar un sistema educativo que coloca a las niñas y a las mujeres en inferioridad. Para conseguir ese objetivo se hace preciso cambiar el marco pedagógico y la dinámica escolar, con el fin de dar cabida a lo cotidiano, al tráfago, a una formación que no vaya encaminada sólo a socializar en el rol profesional. 
Veblen, en La Teoría de la Clase Ociosa, mostró cómo el proceso de subordinación de la mujer al hombre pasaba por generar: a) un modelo educativo diferencial de la agresividad, b) una división de actividades marcada por el género y c) una concepción del gusto diferencial. Es decir, colocó el problema de la construcción social del género en el centro de los procesos de socialización, y, con ello, como una cuestión netamente psicopedagógica. Los mecanismos señalados por Veblen nos dan la pista acerca de qué criterios pueden utilizarse para construir un currículum que no reproduzca estereotipos, que no subordine a la mujer, y que recupere todo un ámbito de lo humano, lo que algunos investigadores han llamado la "subcultura femenina".

La escuela impuso durante mucho tiempo conductas y actividades diferenciales a chicos y chicas, como proyección de concepciones sociales fuertemente arraigadas. Ciertamente hoy esas prácticas han desaparecido, en buena medida, y las que pueden pervivir tienen un carácter residual, pero ello no significa que las actividades no sigan siendo diferenciadas. ¿Queda algo de esa actividad diferencial? ¿Queda algo de esas formas valorativas?. Contestando a la primera pregunta diremos, que las actividades tradicionalmente femeninas han desaparecido de la escuela, lo que significa que, si bien la escuela no las refuerza, tampoco las compensa. Ahora bien, las prácticas diferenciales perviven de modo bien explícito. El trabajo de P. Escario y I. Alberdi ha mostrado que las desventajas de las mujeres en el área de la nuevas tecnologías comienzan en una fase temprana, y que los métodos que emplee la escuela serán importantes para superar este handicap. Recuerdan, siguiendo directrices Europeas, la necesidad de concienciar a la administración educativa de todos los niveles de este hecho para compensarlo. Los planes I y II para la igualdad de oportunidades de la Mujer se han ocupado de ello, pero todavía pervive la tendencia a proyectar las actividades de forma diferencial. ¿Por qué?. Para dar respuesta a esta cuestión tenemos que entrar en la respuesta al segundo de los interrogantes que nos hemos planteado: ¿perviven las formas valorativas negativas?. Muchas de esas formas todavía perviven. Hay acciones, prácticas, cosas, etc., que se siguen considerando femeninas y, por tanto, no valiosas. Es más, cuando se constata en el ámbito de la mujer algo definible como valioso, como puede ser el mejor rendimiento académico de la mujer en todos los niveles educativos, esta ventaja se presenta como una desventaja que reproduce el estereotipo: "Por su mayor sumisión y docilidad, son más constantes, más tranquilas, menos activas, etc.». Se señalan valores femeninos como demérito (Fernández Enguita: 1994). Por tanto, otro criterio que puede señalarse para la selección de contenidos desde una perspectiva sociopolítica, tiene que ver con la selección de actividades y contenidos que rompan la tradicional forma valorativa y recuperen el ámbito de lo cotidiano que boy deja fuera.

En este sentido existen propuestas orientadas a resocializar los comportamientos infantiles en el ámbito tecnológico y profesional (Romero, I.: 1989). Estos dos campos, el tecnológico y el doméstico, tendrán que ser contemplados desde una perspectiva compensatoria, al menos así se está demandando y llevando a la práctica en otros países. En Suecia, Dinamarca e Inglaterra existen experiencias de segre- 
gación a tiempo parcial del currículum (a los niños se les ofrecen actividades de adiestramiento en el rol doméstico y a las niñas se las orienta hacia las tecnologías).

Junto con los dos criterios señalados cabría recordar los propuestos en los dos planes nacionales para la igualdad de oportunidades de la mujer, las orientaciones y normas dictadas por el Ministerio de Educación en materia de coeducación, relativas a los usos lingüísticos, redacción de libros de textos, y recuperación del pasado y la historia de la mujer. Pero creemos que todo ello puede englobarse en un criterio más amplio y generalizador. Es indudable que en los últimos años la mujer ha mejorado su posición en el sistema escolar y percibe la escuela como un ámbito más igualitario que cualquier otro, que le brinda un campo amplio de oportunidades (F. Enguita, 1994), pero la desigualdad sigue existiendo. El problema creemos que se encuentra en la pervivencia de un doble modelo. Un modelo público esencialmente masculino y un modelo privado que puede ser distinto. Las niñas se ven forzadas a socializarse en ese doble modelo y, lo que es peor, así entendidos, los dos roles o modelos son negativos. Este hecho ha llevado a que en diversos países de Europa se hayan puesto en marcha experiencias de currículum segregado y que en España algunos movimientos feministas lo estén reivindicando (Cuadernos de Pedagogía no 171, 1989). No vemos nosotros que ése sea el camino, pensamos que la solución puede estar en un criterio más globalizador, construir la escuela como un espacio que permita investigar otras formas, otros modos, que permitan un ensayo de construcción de lo privado.

\subsection{Igualdad, etnia y cultura}

A medida que se van alcanzando nuevas cuotas de igualdad, que se van alcanzando metas en la construcción de un mundo más justo y más humano, nuevos retos y desafíos educativos se presentan. La existencia de una sociedad cada día más plural, multiétnica y plurilíngüe, ha planteado el desafío de dar respuesta a la multiculturalidad. Esa respuesta en la escuela ha recibido el nombre de intercultura. Afrontar el problema de los estereotipos de raza o etnia en la escuela es una de las cuestiones que incide frontalmente en la selección de los contenidos del currículum. En el caso de España, el problema presenta perfiles muy específicos y de gran complejidad. Somos un país que recibe cada año millones de visitantes, turistas, muchos de los cuales se quedan a residir aquí de forma permanente. En determinadas zonas geográficas esta presencia tiene su reflejo en la escuela y aunque ignoramos la existencia de conflictos, dada la naturaleza específica de esta emigración, puede presentar en un futuro aspectos no tan armónicos. Por otro lado, tradicionalmente España es un lugar de asentamiento de la etnia gitana, lugar de paso y destino de la emigración africana a Europa y receptora de emigrantes de la Europa del este. A todo ello se añade el hecho de ser una comunidad plurilíngüe con los problemas específicos que ello conlleva. Sin duda, todo ello ha planteado problemas en el pasado (construcción del estado de las autonomías), está planteando problemas (debate de las humanidades) y va a seguir planteando problemas. 
No es posible dentro del marco de este trabajo ocuparse de todos y cada uno de los aspectos implicados, por ello hemos adoptado lo que podríamos denominar una perspectiva de máxima generalidad para abordar el problema. ¿Desde la perspectiva sociopolítca es posible dar algún criterio general de selección?. La posibilidad existe siempre que se enfoque, o bien, desde una perspectiva general de la naturaleza humana, -se trataría de ensayar una respuesta desde la filosofía y antropología que, con toda probabilidad, desembocaría en la ética-, o bien, si se determina algún problema que, siendo concreto, se presente con carácter general. Esta segunda perspectiva será la que adoptaremos, seleccionando como problema la cuestión del racismo y la xenofobia. En la respuesta educativa a este problema, probablemente, incluya elementos que sirvan de respuesta a la cuestión general.

Plantearse el problema del racismo y la xenofobia desde un punto de vista educativo supone dar respuesta a una cuestión básica: ¿cómo es el proceso de contacto grupal?. La reflexión sobre los contactos culturales es un reto que debe ser aceptado para poder superar los tres obstáculos que con más frecuencia aparecen al tratar esta cuestión. La tesis que niega el mestizaje cultural, la tesis que afirma que las culturas tienen una esencia sagrada, inamovible e inmutable a través del curso del tiempo y la tesis del relativismo cultural absoluto. La segunda tesis es un corolario de la primera, y la tercera funciona como condición de posibilidad de las otras dos.

Para dar respuestas cumplidas a estas cuestiones sería preciso que nos ocupáramos de un conjunto de problemas éticos que el racismo presenta dentro de las ciencias sociales, y que agrupados y sucintamente enunciados serían:

a) ¿Es el racismo y la xenofobia un comportamiento innato o adquirido?. ¿Está inscrito en la naturaleza humana?. ¿Son de carácter grupal o individual?.

b) Si, como sospechamos, es un comportamiento aprendido vinculado a la diferenciación etnosemántica y cultural entre nosotros-ellos, ¿qué factores psicosociales y cognitivos favorecen los contactos conflictivos, o, por el contrario, los evitan?.

c) ¿Cómo se han producido los contactos intergrupales?. ¿Cuál es la historia?. ¿Hay pautas diferenciales que nos permitan plantear la convivencia de forma más ética?.

d) ¿Es posible el mestizaje cultural?.

No es posible aquí ocuparse del desarrollo que estas cuestiones plantean, pero sí adelantar tres tesis que defendemos y que pueden funcionar como criterios sociopolíticos de selección de los materiales curriculares.

1) Si queremos afrontar con racionalidad la construcción de una cultura multiétnica, es necesario afirmar, aunque pueda parecer obvio, la existencia de una naturaleza humana común. Para el relativismo cultural el hombre no tiene naturaleza, sino que es un ser infinitamente maleable por la cultura. Un individuo no es otra cosa, en el fondo, que un síntoma de lo que los demás piensan de él. 
2) Frente a las posiciones que defienden un relativismo cultural a ultranza como única respuesta educativa a una realidad multicultural, proponemos un relacionismo pluralista que supone aceptar que todo el mundo (sea individuo o cultura) se halla (quiéralo o no y aunque quiera no estarlo) en una determinada posición (perspectiva, ángulo, partido, etc.) relativa o relacionada con las de los que en el mismo espacio toman otras posiciones (individuales o culturales).

3) La única respuesta posible a la existencia de una multiplicidad de culturas, es aceptar la posibilidad de existencia de un "mestizaje de culturas" que desarrollen y potencien esa naturaleza común.

\section{CRITERIOS PSICOLÓGICOS}

\subsection{La psicología como fuente del currículum}

Ya Tyler (1949), un primer referente de la teoría curricular, establecía la psicología como una de las fuentes del currículo y nadie cuestiona hoy su papel en este contexto, un papel en todo caso complementario de las aportaciones de la base epistemológica, sociológica y pedagógica. También es cierto que la fuente psicológica no proporciona prioritariamente criterios para la selección de contenidos sino para su organización y secuenciación así como pautas para establecer los métodos y actividades pertinentes que propicien su aprendizaje. Es éste un tema muy poco abordado en la literatura sobre el currículum, de modo que nuestra aproximación será tentativa y provisional. Los criterios que propondremos derivan de los campos de la psicología citados clásicamente cuando se analizan las relaciones entre psicología y currículum: psicología evolutiva y del desarrollo y psicología del aprendizaje y de la instrucción (Coll, 1987; Gimeno, 1981; Gimeno y Pérez Gómez, 1992; Taba, 1974; Tyler, 1949).

4.2. Criterios de selección y organización derivados de la psicología evolutiva y del desarrollo

Un primer criterio, muy general, coherente con cualquier psicología del desarrollo, podría ser el siguiente:

Capacidad para propiciar el desarrollo integral del alumno. Se trata de seleccionar los contenidos que permitan trabajar todas las dimensiones humanas: cognitiva, afectiva, conductual, relacional, etc. Este criterio subraya la necesidad de incluir en el currículum diversidad de contenidos que cubran la integridad del desarrollo humano. Algunos de estos contenidos han sido incorporados explícitamente al currículum oficial español por la reforma educativa. Sin embargo, hay otros contenidos sumamente relevantes no incluidos tan explícitamente en el currí- 
culum oficial, como los modos de interacción, habilidades sociales, hábitos, etc. que también deben ser cubiertos en la enseñanza escolar.

Este primer criterio, muy general, debe ser completado con otros que ayudan a su operativización. El primero de los que siguen es coherente con las aportaciones de la teoría psicogenética y evolutiva de Piaget y de la escuela de Ginebra:

Adecuación al desarrollo cognitivo del sujeto que aprende. Un sujeto sólo puede procesar la información que es capaz de asimilar de acuerdo con sus estructuras cognitivas, por lo que el nivel de complejidad de los contenidos que se presenten al sujeto ha de ajustarse a su desarrollo cognitivo. Si en esto el acuerdo es general, los límites de lo que se puede aprender ya no están tan claros. Así, por ejemplo, los trabajos de estimulación temprana permiten lograr aprendizajes en edades más bajas de lo que tradicionalmente se pensaba y de lo que prescriben los currículos escolares. No se cuestiona la invarianza del desarrollo -las etapas evolutivas se recorren unas después de otras, apoyándose cada una en la anteriorpero sí los límites de edad, que parecen hoy mucho más flexibles de lo que postulara Piaget. El mismo Bruner (1984 y 1988) afirma que se puede enseñar cualquier materia a cualquier niño a cualquier edad si se hace de forma "honesta" (con la metodología adecuada), lo que es una auténtica provocación pedagógica y una aparente ruptura con el planteamiento piagetiano. Así, el autor ejemplifica cómo se puede enseñar ecuaciones de segundo grado a niños de 8 años. Evidentemente, el procedimiento no es el procedimiento abstracto y formal que se utiliza en $2^{\circ}$ de la ESO, sino un procedimiento intuitivo y manipulativo. Por eso, los postulados piagetianos deben ser complementados con las aportaciones de la psicología soviética, específicamente de Luria, Vygotsky y Leontiev, desarrolladas posteriormente por Wertsch, Cazden, etc., más posibilistas desde el punto de vista educativo, que desembocan en el enfoque sociocultural, que entiende de una manera diferente las relaciones entre aprendizaje y desarrollo -en Piaget la primacía era del desarrolloy conceden una gran importancia al contexto, a las relaciones interpersonales y a los procesos de mediación del educador. Si para Piaget el buen aprendizaje es el que se subordina al desarrollo, para Vygotsky el buen aprendizaje es el que "tira" del desarrollo, el que se sitúa en la Zona de Desarrollo Próximo, que es la que media entre el desarrollo potencial -lo que el niño puede hacer con ayuda de un mediador- y el desarrollo real o desarrollo efectivo -lo que el niño puede hacer por sí sólo, de manera autónoma-. En este contexto se ubica la metáfora del "andamiaje" de Bruner, que es la ayuda que se presta y se retira cuando el aprendiz consigue la competencia suficiente para funcionar con autonomía. Un criterio que se puede derivar de estos planteamientos, complementario del anterior, es el siguiente:

Capacidad para suponer un reto cognitivo. Se trata de seleccionar contenidos -y objetivos y actividades- cuyo aprendizaje suponga un reto, pero un reto posible para los aprendices, ubicándose en la Zona de Desarrollo Próximo, contenidos que "tiren" del desarrollo, yendo un poco por delante. Se puede defender la exis- 
tencia de unos "universales cognitivos" -capacidades cognitivas básicas: capacidad para formar conceptos, para razonar lógicamente, para generalizar, etc.-, comunes a todos los seres humanos. Sin embargo la puesta en práctica de esas capacidades cuando nos enfrentamos a tareas concretas de solución de problemas es diferente, y la diferencia depende de los aprendizajes específicos y de las experiencias educativas. Ahí es donde tiene sentido la intervención educativa que plantee retos, retos que ayuden a ir más allá, a progresar en el desarrollo y uso de la competencia cognitiva.

En este criterio se observa claramente la necesidad de tener en cuenta conjuntamente los contenidos y la forma de trabajar con ellos, así como los objetivos educativos y las competencias que se quieren desarrollar.

Por otra parte, la adecuación al desarrollo, a la hora de diseñar los curricula escolares tropieza con una clara dificultad: los diferentes ritmos de aprendizaje de los alumnos, evidentes en una escuela comprensiva como la española en la enseñanza obligatoria. De ahí que sea necesaria establecer otro criterio que completa los anteriores:

Diversificación. Este criterio implica establecer diferentes niveles de contenidos en dificultad y complejidad, que respondan a las necesidades de "todos" los alumnos y no sólo a las de un grupo ideal que no existe. La reforma educativa española, a nivel legal, cubre estas necesidades a través de la atención a la diversidad, con mecanismos como las adaptaciones curriculares y la diversificación curricular, lo que no implica que su puesta en práctica no tropiece con dificultades por la complejidad del tema, por la falta de formación de los profesores y de recursos personales y materiales.

4.3. Criterios derivados de la psicología del aprendizaje y de la psicología de la instrucción

Las teorías del aprendizaje han ido cobrando fuerza en la determinación del currículum desde las primeras formulaciones de Tyler (1949) y su importancia en este ámbito es hoy incuestionable. Desde muy atrás se puede rastrear su influencia en el currículum (Taba, 1974). Así, la teoría de la disciplina mental o de las facultades, que entiende que la mente está integrada por facultades que se desarrollan con el ejercicio y defiende que determinadas materias, especialmente las difíciles, como el Latín o las Matemáticas, enseñan a razonar. En este planteamiento se da prioridad al "orden lógico" de las disciplinas al que el alumno debe adaptarse. También el conductismo ha ejercido notable influencia en la presentación de los contenidos curriculares. Los objetivos operativos, parcelados al máximo, la determinación de conductas mensurables, el uso del refuerzo de cada paso correcto, el recitado y la repetición, el análisis de tareas o la modificación de conducta son derivaciones de esta teoría. 
Nosotros, sin desdeñar las aportaciones positivas del conductismo, nos decantamos por una psicología del aprendizaje cognitivista y constructivista. Realizaremos, en primer lugar, una síntesis de los postulados de estas teorías para extraer criterios de selección y secuenciación de contenidos y, posteriormente, haremos lo propio con las principales teorías de la transferencia, aspecto importante dentro de la psicología del aprendizaje y de la instrucción.

\subsubsection{Cognitivismo}

El cognitivismo, cuya fecha oficial de nacimiento se fija en 1956 (Gardner, 1988), año del Simposium sobre teoría de la información en el Instituto de Tecnología de Massachusetts, se plantea como alternativa al conductismo. La explicación de la conducta se refiere a estados mentales -intenciones, significados, etc.- y se enfatiza el estudio, descripción y explicación de los procesos mediacionales. El aprendizaje se entiende como cambio cognitivo no producido exclusivamente por asociación, sino por reestructuración y se defiende el constructivismo: las representaciones de la realidad son construidas por el sujeto y siempre se conoce a partir de lo ya conocido, de modo que la realidad se interpreta a la luz de los esquemas mentales disponibles (Aznar, Barrón y Gargallo, 1997). Se recupera el papel del sujeto como controlador de la conducta y de sí mismo y se considera la conducta humana como totalidad.

Hay dos grandes líneas en la psicología cognitiva (Pozo, 1990): la teoría del procesamiento de la información y la psicología cognitiva europea, que se suele denominar constructivismo.

\subsubsection{La teoría del procesamiento de la información}

Esta teoría, en la que destacan los trabajos de Adams, Anderson, Atkinson, Mayer, Newell, Simon, Pascual-Leone, etc. considera al hombre como "informívoro", como procesador activo de información, que recibe información del medio, la transforma, la hace propia y actúa de acuerdo con ella. Ello implica la superación del esquema simplista E-R del asociacionismo, ya que el ser humano "lee" el estímulo, interpreta la realidad de acuerdo con sus esquemas cognitivos y responde de acuerdo con su percepción de la realidad, no de manera automática, frente al estímulo.

Los modelos de procesamiento de la información -que utilizan la metáfora del ordenador para representar los procesos cognitivos del ser humano- integran una serie de estructuras (Atkinson y Shiffrin, 1968): registros o receptores sensoriales, memoria a corto plazo, memoria a largo plazo, y efectores o generadores de respuesta, que soportan los procesos que moviliza el sujeto para procesar la información: procesos atencionales, de codificación, de retención y de recuperación. Mediante ellos el sujeto selecciona la información, la elabora, la organiza, la hace 
propia, la almacena y la recupera. Las estrategias de aprendizaje son los mecanismos de control que utilizamos para dirigir y modular esos procesos, que son nuestros procesos de aprendizaje.

Un criterio de selección de contenidos que se puede derivar de la teoría del procesamiento de la información, consecuente con sus postulados, es:

Aprendizaje estratégico. Este criterio implica trabajar la metacognición, término acuñado por Flavell (1977) para referirse al conocimiento y control de los propios procesos cognitivos, de enseñar a "aprender a aprender". Las estrategias de aprendizaje son, pues, contenidos educativos procedimentales que hay que incluir en el currículum. Una propuesta de contenidos de esta índole debería integrar (Aznar, Barrón y Gargallo, 1997): estrategias afectivo-motivacionales y de control del contexto, estrategias de búsqueda, selección y recogida de información, estrategias de procesamiento propiamente dichas, dirigidas al aprendizaje de los materiales, y estrategias metacognitivas -referidas al conocimiento y control de los propios procesos cognitivos y de las habilidades y destrezas en función del contexto, de las demandas de la tarea y de los objetivos a lograr; las clásicamente propuestas en el ámbito del control son la planificación, el control/regulación y la autoevaluación de la propia actividad-. También supone incluir estrategias de solución de problemas, una orientación clásica en la ciencia cognitiva. Se trataría de lograr que los alumnos, al dejar la escolaridad obligatoria, hubieran logrado un cierto nivel de conocimiento experto (Vázquez, 1991) en aprender a aprender y en solucionar problemas.

Estos contenidos, indicados para favorecer el aprendizaje estratégico, deben ser presentados pedagógicamente de modo que al trabajar con ellos el alumno adquiera conocimientos, aprenda a usar y comprender procedimientos y desarrolle actitudes y sentimientos positivos hacia ellos. Como ocurre con cualesquiera otros contenidos, éstos, una vez trabajados, deberían convertirse en resultados del aprendizaje, en nuevas o mejores competencias y, por lo tanto, en parte del desarrollo integral de la personalidad.

\subsubsection{El constructivismo}

El constructivismo, a nivel psicológico, se fundamenta en las elaboraciones de pioneros como la Gestalt o Bartlett, padre de la teoría de los esquemas, y en las de figuras clave como Vygotsky, Piaget, Ausubel, con aportaciones de Wallon, Kelly y Bruner, entre otros, y no presenta hoy una teoría global y unitaria que pueda dar cuenta de todos los procesos de aprendizaje, aunque oferta claves explicativas de suma relevancia para la intervención educativa. Las teorías de los diferentes autores presentan coincidencias sustantivas en aspectos básicos y también divergencias importantes -ya se han comentado brevemente las diferencias existentes entre Piaget y Vygotsky en lo referente a relaciones aprendizaje/desarrollo-. El conocimiento se entiende no como copia de la realidad sino como construcción del ser 
humano. Desaparece la noción objetivista de verdad como correspondencia entre la mente y la realidad para pasar a hablar de ajuste progresivo, de procesos de negociación e interacción que progresivamente permiten acercarse a esa realidad de manera cada vez más segura. El conocimiento es un proceso dinámico e interactivo por medio del cual se interpreta la realidad construyendo cada vez modelos explicativos más potentes. Conocemos siempre a partir de lo ya conocido y elaboramos significados partiendo de lo que ya sabemos.

Consideramos que las tres teorías más potentes por su nivel explicativo y por sus repercursiones educativas son la teoría psicogenética de Piaget, que integra su teoría del desarrollo, su teoría de la inteligencia y su modelo de la equilibración y su apuesta por el aprendizaje por descubrimiento, la teoría de la asimilación cognoscitiva y del aprendizaje significativo de Ausubel, y el enfoque sociocultural y la teoría de la Zona de Desarrollo Próximo de Vygotsky, que han sido desarrollados, aparte de por los discípulos antes citados, también por Bruner, el genial psicólogo americano.

Proponemos, a continuación, un criterio general de selección y secuenciación de contenidos que se puede derivar de las teorías constructivistas:

Significatividad y reconstrucción. Los contenidos han de ser seleccionados y presentados de modo que se favorezca el aprendizaje significativo y la construcción personal de significados. Para hacer operativo este criterio general proponemos otros que lo concretan:

Significatividad lógica. Los contenidos han de estar bien estructurados. El material debe ser potencialmente significativo, no arbitrario ni confuso. No pocos libros de texto, utilizados como soporte de los contenidos, presentan lagunas en este sentido, por falta de coherencia en la presentación de los contenidos, por inadecuada estructuración, etc.

Este criterio hace referencia a dos aspectos indisolublemente unidos al contenido propiamente dicho: la claridad lógica y la forma de organización, relación o estructuración entre unidades de contenido. Ambos aspectos son esenciales desde el punto de vista de la calidad pedagógica del propio contenido.

Significatividad psicológica. La selección y presentación de los contenidos ha de adecuarse a los conocimientos ya adquiridos por el alumno, que debe disponer en su estructura cognitiva de elementos pertinentes y relacionables para integrar los nuevos contenidos.

Partir de lo ya conocido, de lo inmediato, de la experiencia del alumno. La selección y estructuración de los contenidos y la presentación de los mismos en los libros de texto, en nuestro país, al menos en la educación infantil y primaria, suele partir de la experiencia inmediata del alumno, para ir ensanchando la perspectiva, progresivamente, a modo de círculos concéntricos. Sin embargo, si no se tiene cuidado, este criterio puede llevarse a un cierto reduccionismo. Así, no es extraño que nuestros alumnos de primaria e incluso de inicios de la secundaria, queden excesivamente "pegados" a lo inmediato. Pueden conocer a fondo la geografía e histo- 
ria de su comunidad y localidad, pero no la de España, Europa o el mundo. Por eso, este criterio debería ser complementado con otro, también constructivista:

Uso del contraste y de experiencias diferentes de las propias. Se trata de ofertar contenidos, experiencias y realidades nuevas que sirvan de contraste con las que ya tienen los alumnos de su entorno inmediato. Un ejemplo ilustrativo del uso de este criterio es el currículum de humanidades de Bruner (1988), desarrollado para alumnos de 10 años de enseñanza primaria, en que, de manera experiencial e intuitiva, se abordan una serie de elementos culturales fundamentales en el desarrollo humano: el lenguaje, la fabricación de herramientas, la organización social, la crianza y educación del niño y la visión el mundo. Para trabajar estos bloques se dispone de materiales filmados sobre la vida de dos sociedades de cazadores-recolectores, los esquimales netsilik y los bosquimanos del desierto del Kalahari, y de dos grupos de primates, macacos y babuinos. Los niños pueden contrastar los modos de resolver su vida de estos seres humanos y primates entre sí y frente a su propio modo de vida, en la sociedad estadounidense desarrollada. El curso dispone de materiales diversos que incitan a los alumnos a formular conjeturas e hipótesis y a comprobarlas. La idea fundamental que Bruner quiere que los niños descubran es que "todo hombre es 'humano', con independencia de lo avanzada o 'primitiva' que sea su civilización" (Bruner, 1988: pp. 183). El autor no cuestiona que puede ser recomendable iniciar la enseñanza de las ciencias sociales en el estudio del ambiente inmediato, pero estima que este planteamiento cae en el defecto de que difícilmente se puede captar la generalidad en las cosas familiares. La manera de lograrlo, según él, es a través del análisis de otras realidades y modos de afrontar los problemas comunes a los seres humanos.

Promover conflictos y disonancias cognitivas. Este criterio alude a la pertinencia de seleccionar contenidos e información disonante que choque con las preconcepciones inadecuadas de los niños y de proponer actividades que supongan un problema para los alumnos -vgr. cómo respiran los peces debajo del agua, por qué un cubito de hielo se derrite antes envuelto en papel de aluminio que en una bufanda, por qué flotan los barcos o vuelan los aviones a pesar de su peso, por qué unos países son ricos y otros pobres, etc.-, de modo que se propicie el conflicto cognitivo y se rompa el equilibrio para provocar, por procesos de asimilación y acomodación, la reestructuración cognitiva y el logro de estadios superiores de equilibrio.

Ofertar "espacios" posibles para la actividad. Se trata de que la oferta curricular no se dé completamente terminada y elaborada sólo para ser memorizada. El alumno ha de tener oportunidades de actuar sobre los contenidos: completar, corregir, cuestionar, elaborar, ampliar, etc.

Los contenidos, como saberes y productos culturales que son, requieren ser comprendidos, más que como resultados acabados y cerrados, como resultados provisionales fruto de un proceso histórico de búsqueda de la verdad, o si se prefiere, de interpretación de la realidad. Por ello, este criterio, más que incidir en el 

CRITERIOS DE SELECCIÓN DE LOS CONTENIDOS DEL CURRÍCULUM

problema de la selección, nos recuerda que los resultados del aprendizaje dependen tanto de los contenidos como de la forma en que se trabajen, por lo que es necesario que el educador tenga muy presente qué objetivos pretende que sus alumnos alcancen, porque un mismo contenido puede dar lugar a logros muy distintos $\mathrm{y}$, a veces, hasta contrapuestos.

\subsubsection{La transferencia}

La transferencia es uno de los principios claves de la educación. La educación escolar tiene sentido en tanto en cuanto prepara para la vida y en tanto en cuanto lo aprendido puede utilizarse en otros contextos.

En una breve síntesis de las teorías de la transferencia (Ausubel, 1990; Beltrán, 1987 y 1993; Castillejo, 1981; Montané, 1984) encontramos la teoría más antigua, la de la disciplina mental o de las facultades, ya comentada, que defiende una transferencia general, automática e ilimitada de las facultades desarrolladas por el ejercicio en las materias más difíciles, que se pueden aplicar luego en cualquier aprendizaje. Esta teoría fue desacreditada por William James en su famoso experimento de la memorización y por Thorndike (1901). Éste último formula en 1906 la teoría de los elementos idénticos, que defiende que la transferencia ocurre cuando se dan los mismos elementos en la situación de enseñanza y en sus aplicaciones. Esta teoría influyó en la elaboración de currículos más relevantes que trabajaban las habilidades instrumentales y los contenidos más esenciales, como por ejemplo la ortografía, la aritmética o el vocabulario básico. Presenta una limitación clara, que es la imposibilidad de enseñar en la escuela todos los conocimientos, habilidades y destrezas que un alumno necesitará para su vida, especialmente en sociedades dinámicas y cambiantes. Una teoría coetánea es la de la generalización de Judd (1908), para el que la clave está en la comprensión y generalización de los principios subyacentes a los hechos. Se trata de ayudar al alumno a extraer los conceptos centrales y esenciales y los principios generales. La generalización será más potente si los principios que se trabajan tienen aplicación fuera de la clase. Dejando de lado la teoría de la transferencia del condicionamiento operante de Skinner encontramos la teoría de las habilidades o del aprender cómo aprender de Harlow (1949). Esta teoría defiende la pertinencia de trabajar más las habilidades que la información fáctica, que puede encontrarse cuando se necesite. También Ausubel (1990) desarrolla una teoría de la transferencia, es la teoría de la estructura cognitiva, que defiende que el aprendizaje significativo modifica la estructura cognitiva existente y afecta a aprendizajes posteriores, produciendo necesariamente transferencia, que se ve facilitada en la medida en que los aprendizajes anteriores han dado como resultado una estructura cognitiva caracterizada por ideas bien desarrolladas, estables e inclusivas a las que puedan vincularse materiales nuevos y potencialmente significativos.

Un cierto eclecticismo integrador puede ser lo adecuado, integrando los valores de la teoría de la generalización de los principios y la pertinencia de trabajar de 
modo adecuado las habilidades y estrategias, sin desestimar la relevancia del aprendizaje significativo de Ausubel y los efectos positivos del uso, en determinados momentos, de los elementos idénticos. A partir de ahí se pueden derivar los siguientes criterios:

Seleccionar y secuenciar los contenidos y articular los métodos de modo que se favorezca lo más posible la transferencia. Este primer criterio, muy general, se puede operativizar a través de otros tres, complementarios:

Relevancia. Funcionalidad. Utilidad para la vida. Adecuación al contexto y a las necesidades sociales. Se trata de seleccionar contenidos importantes, útiles para la vida, de modo que las materias contengan el mayor número de similitudes entre lo que se aprende y las situaciones vitales de aplicación de lo aprendido.

Aprendizaje de principios. Se trata de seleccionar las ideas básicas de cada materia, las que integran su estructura fundamental y ofertarlas en los primeros cursos de manera simplificada para, progresivamente, ofrecer formas más complejas, procediendo de lo concreto a lo abstracto, de lo específico a lo general. Un ejemplo de este planteamiento es el currículum "en espiral" de Bruner, que permite retornar sobre los contenidos con progresiva profundidad. En todo caso, la transferencia no es automática y la clave está tanto en contenidos adecuados como en métodos pertinentes. La transferencia depende tanto de lo que se aprende como del modo como se aprende. Si se ayuda al estudiante a descubrir por sí mismo los principios de manera inductiva los comprende más claramente, los retiene mejor y los emplea más eficazmente. Hay que tener en cuenta que el niño de la escuela primaria se encuentra en el estadio de operaciones concretas y necesita una notable experiencia directa antes de que pueda abstraer o aplicar conceptos significativos. Por eso hay que vincular los aprendizajes con la vida real. Como no siempre se puede utilizar el aprendizaje por descubrimiento, es imprescindible, en cualquier caso, lograr el aprendizaje significativo y es fundamental, para potenciar la transferencia, ejemplificar su uso y enseñar la forma de aplicar los principios y conceptos en situaciones diversas.

Aprendizaje de habilidades instrumentales. Es fundamental consolidar, en los primeros aprendizajes, una serie de destrezas básicas e instrumentales, como la lectoescritura, el cálculo, la expresión oral y el dominio de las técnicas de estudio y estrategias de aprendizaje -es una habilidad instrumental necesaria, también, el manejo de la red informática-. Se trata de aprendizajes sumamente transferibles, que se utilizan en las diversas materias y en la vida cotidiana, y que son necesarios para seguir aprendiendo y para manejarse en la sociedad.

Establecer prioridades respecto a los contenidos que merecen ser enseñados $\mathrm{y}$ aprendidos en las instituciones educativas, se ha convertido en una necesidad de primer orden. A lo largo de estas páginas hemos intentado ofrecer criterios para fundamentar y emprender esa tarea desde una perspectiva pedagógica en la que están implicadas cuestiones que afectan al problema epistemológico de la construcción y organización del conocimiento, a las necesidades sociales y las decisio- 
nes políticas, y a las exigencias que plantean los procesos de aprendizaje y desarrollo del sujeto. Cada una de estas cuestiones constituyen fuentes de selección de criterios y contenidos que por sí mismas resultan insuficientes sin una perspectiva pedagógica integradora. Finalmente hay que destacar la importancia de la puesta en práctica real de las propuestas curriculares que corresponde a los profesores, lo que reclama una formación pedagógica que supere el reduccionismo de confundirla con el dominio de las materias.

\section{BiBLIOGRAFÍA}

APPLE, M. W. (1996): Política cultural y educación. Morata. Madrid.

ARGOS, J. Y EZQUERRA, Mà . P. (1999): Principios del curriculum. IV Jornadas de Teorias e Instituciones Educativas Contemporáneas. Servicio de Publicaciones de la Universidad de Cantabria. Santander.

ATKINSON, R. C. y SHIFFRIN, R. M. (1968): Human memory; a proposed system and its control processes. En SPENCE y SPENCE (Eds.), The psychology of learning and motivation. Academic Press. Nueva York.

AUSUBEL, D. P.; NOVAK, J. D. y HANESIAN, H. (1990): Psicología educativa: un punto de vista cognoscitivo. Trillas. Méjico.

AZNAR, P.; BARRÓN, A. y GARGALLO, B. (1997): Tecnología educativa y procesos de aprendizaje. En BARROSO, C. y GALLARDO, M. (Coord.): Tecnologías y formación permanente. Universidad de la Laguna. La Laguna.

BALANDIER, G. (1956): Race relations in world perspectivs. Honolulu. University of Hawai Press. BELTRÁN, J. (1987): Psicología de la educación. Eudema. Madrid.

- (1993): Procesos, estrategias y técnicas de aprendizaje. Síntesis. Madrid.

BENAVOT, A. y otros (1991): «El conocimiento para las masas: modelos mundiales y curricula nacionales". Revista de Educación, 295, 317-344.

BERGER, P. y LUCKMANN, Th. (1991): La construcción social de la realidad. Amorrortu Editores. Buenos Aires.

BERNSTEIN, B. (1971): On the Classification and Framing of Educational Knowledge. En YOUNG, M. (Edit.): Knowledge and Control: New Directions for the Sociology of Education, Londres, Collier - MacMillan.

- (1996): Pedagogía, control simbólico e identidad. Morata. Madrid.

BLANCO, N. (1994): “Los contenidos del currículum». En ÁNGULO, J. F. y BLANCO, N. (Coords.): Teoria y desarrollo del curriculum. Ediciones Aljibe. Málaga, pp. 233-262.

BONFIL, G. (1987): Mexico Profundo. México. CIESAS.

BOURDIEU, P. y GROS, F. (1990): “Principios para una reflexión sobre los contenidos de la enseñanza". Revista de Educación, 292, 417-424.

BRUNER, J. (1984): Acción, pensamiento y lenguaje. Alianza. Madrid.

- (1988): Desarrollo cognitivo y educación. Morata. Madrid.

CASTILLEJO, J. L.; ESCÁMEZ, J. y MARÍN, R. (1981): Teoría de la educación. Anaya/2. Madrid.

CLEMENTE LINUESA, M. (1995): “Seleccionar contenidos: opción cultural o decisión técnica”. Enseñanza. Anuario Interuniversitario de Didáctica, 13, 261-274.

CLEMENTE, L.; DOMÍNGUEZ GUTIÉRREZ, A. B. y RAMÍREZ ORELLANA, E. (1999): “Cómo seleccionan los profesores los contenidos en los proyectos curriculares de centro en la 
JULIO VERA VILA, ÁNGEL GARCÍA DEL DUJO, JOSÉ V. PEÑA CALVO Y BERNARDO GARGALLO LÓPEZ CRITERIOS DE SELECCIÓN DE LOS CONTENIDOS DEL CURRÍCULUM

Comunidad Autónoma de Castilla y León". Revista de Estudios del Currículum, vol. 2, no $1,161-188$.

COLL, C. (1987): Psicología y currículum. Paidós. Barcelona.

- y POZO, J. I.; SARABIA, B. y VALLS, E. (1992): Los contenidos en la reforma. Enseñanza $y$ aprendizaje de conceptos, procedimientos y actitudes. Santillana. Madrid.

COLOM, A. J. (1987): La educación como sistema. En CASTILlEJO, J. L.; COLOM, A. J. y OTROS: Pedagogía Sistémica. Ceac. Barcelona, pp. 83-107.

- (1994): "El currículum escolar". En CASTILLEJO, J. L.; VÁZQUEZ, G.; COLOM, A. J. y SARRAMONA, J.: Teoría de la Educación. Taurus. Madrid, pp. 83-112.

- y MELICH, J.C. (1994): Después de la modernidad. Nuevas filosofías de la educación. Paidós. Barcelona.

ESCÁMEZ SÁNCHEZ, J. (1987): Antropología del currículum. En SARRAMONA LÓPEZ, J. (Ed.): Curriculum y educación. Ediciones Ceac. Barcelona, pp. 25-44.

ESCARIO, P. y ALBERDI, Y. (1987): El impacto de la Nuevas Tecnologías en la Formación y el Trabajo de las mujeres. Madrid. Instituto de la Mujer.

ESTEVE, J. M.; VERA, J.; TERRÓN, J.; FRANCO, S. y CIVILA, A. (1999): Cultura, cultura escolar y contenidos de enseñanza. En ARGOS, J. y EZQUERRA, Ma. P.: Principios del curriculum. IV Jornadas de Teorias e Instituciones Educativas Contemporáneas. Servicio de Publicaciones de la Universidad de Cantabria. Santander, pp. 119-134.

FLAVELL, J. H. y WELLMAN, H. M. (1977): Metamemory, en KAIL, R. K. y HAGEN, J. W. (Eds.): Perspectives on the development of memory and cognition. N. J. Erlbaum. Hillsdale.

GARCÍA CARRASCO, J. y GARCÍA DEL DUJO, A. (1996): Teoria de la Educación. Vol. I. Educación y acción pedagógica. Ediciones Universidad. Salamanca.

GARDNER, H. (1988): La nueva ciencia de la mente. Paidós. Barcelona.

GIMENO SACRISTÁN, J. (1981): Teoría de la enseñanza y desarrollo del currículo. Anaya/2. Madrid.

- (1988): El currículum: una reflexión sobre la práctica. Morata. Madrid.

- (1998): Poderes inestables en educación. Morata. Madrid.

- y PÉREZ GÓMEZ, A. I. (1992): Comprender y transformar la enseñanza. Morata; Madrid. HARLOW, H. F. (1949): "The formation of learning sets". Psychological Review, $\mathrm{n}^{\circ} 56$.

HIRST, P. H. (1974): Knowledge and the Curriculum. Routledge and Kegan Paul. Londres.

IBÁÑEZ-MARTÍN, J. A. (1987) El problema del contenido del currículo: un primer acercamiento desde la Filosofía de la Educación. En SARRAMONA LÓPEZ, J. (Ed.): Currículum y educación. Ceac. Barcelona, pp. 11-24.

JUDD, C.H. (1908): "The relation of special training to general intelligence». Educational Review, $\mathrm{n}^{\circ} 36$, pp. 28-42.

KEMMIS, S. (1988): El currículum. Más allá de la teoría de la reproducción. Morata. Madrid.

LAWTON, D. (1989): Education, culture and the national curriculum. Hodder and Stoughton. London.

MINISTERIO DE EDUCACIÓN Y CIENCIA (1989): Diseño curricular base: educación infantilleducación primaria/educación secundaria. MEC. Madrid.

MEYER, J. y otros (1992): School knowledge for the masses. World models and national primary curricular categories in the twentieth century. Falmer Press. Lewes.

MONTANÉ, J. (1984): Aportaciones de la transferencia de los aprendizajes a la psicología de la educación. Aspectos teóricos-prácticos. En SANVISENS, A. y otros: Introducción a la pedagogia. Barcanova. Barcelona.

MORIN, E. (1984): Ciencia con consciencia. Anthropos. Barcelona.

(C) Ediciones Universidad de Salamanca

Teor. educ. 11, 1999, pp. 13-52 
JUIO VERA VILA, ÁNGEL GARCÍA DEL DUJO, JOSÉ V. PEÑA CALVO Y BERNARDO GARGALLO LÓPEZ

CRITERIOS DE SELECCIÓN DE LOS CONTENIDOS DEL CURRÍCULUM

- (1994): Introducción al pensamiento complejo. Gedisa. Barcelona.

NORBERT, E. (1994): Conocimiento y poder. Ediciones de La Piqueta. Madrid.

ORTEGA Y GASSET, J. (1975): La misión de la universidad y otros ensayos. Revista de Occidente, Madrid. $6^{\mathrm{a}}$ ed.

PÉREZ GÓMEZ, A. (1988): Currículum y enseñanza: Análisis de componentes. Universidad de Málaga. Málaga.

- (1998): La cultura escolar en la sociedad neoliberal. Morata. Madrid.

PINEAU, P. (1999): "Premisas básicas de la escolarización como construcción moderna que construyó la escolaridad». Revista de Estudios del Currículum, vol. 2, ํㅜㄹ 1, 38-61.

POPKEWITZ, Th. (1987): "La producción del conocimiento escolar y los lenguajes curriculares". Revista de Educación, 282, 61-85.

- (1994): "Política, conocimiento y poder: algunas cuestiones para el estudio de las reformas educativas". Revista de Educación, 305, 103-137.

POSTMAN, N. (1994): Tecnópolis. La rendición de la cultura a la tecnología. Galaxia Gutemberg/Círculo de Lectores. Barcelona.

POZO, J. I. (1990): Teorias cognitivas del aprendizaje. Morata. Madrid.

PUELLES BENÍTEZ (DE) M. (1999): Principios sociopolíticos del currículum. En ARGOS, J. Y EZQUERRA, M. P. (Ed.): Principios del Currículum. IV Jornadas de teorias e Instituciones Contemporáneas de Educación. Servicio de Publicaciones de la Universidad de Cantabria. Santander, pp. 101-118.

SARRAMONA LÓPEZ, J. (Ed.) (1987): Currículum y educación. Ceac. Barcelona.

- (1989): Los contenidos del currículum. En ESTEVE ZARAZAGA, J. M. (Ed.): Objetivos y contenidos de la educación para los años noventa. Universidad de Málaga, pp. 37-54.

- (1999): Principios Pedagógicos del Currículum. En ARGOS, J. Y EZQUERRA, Ma․ P.: Principios del curriculum. IV Jornadas de Teorias e Instituciones Educativas Contemporáneas. Servicio de Publicaciones de la Universidad de Cantabria. Santander, pp. 79-100.

SAVATER, F. (1997): El valor de educar. Ariel. Barcelona. $7^{\mathfrak{a}}$ ed.

- (1999): Potenciar la razón. En AA.VV.: La educación que queremos. Santillana. Madrid, pp. 55-71.

SCHNITMAN, D. F. (1994): Nuevos paradigmas, cultura y subjetividad. Paidós. Barcelona.

TABA, H. (1974): Elaboración del currículo. Troquel. Buenos Aires.

THORNDIKE, E. L. y WOODWORTH, R. S. (1901): "The influencie of improvement in one mental function upon the efficiency of other functions". Psychological Review, $\mathrm{n}^{\circ} 8$.

- (1906): The principles of teaching. A.G.Seiler. Nueva York.

TORRES, J. (1994): Globalización e iterdisciplinariedad: el curriculum integrado. Morata. Madrid.

TRILLA, J. (1985): La educación fuera de la escuela. Planeta. Barcelona.

TYLER, R.W. (1949): Principios básicos del currículum. Troquel. Buenos Aires.

VÁZQUEZ GÓMEZ, G. (1985): Currículum oculto y manifiesto. En AA.VV.: Condicionamientos sociopolíticos de la educación. Ceac. Barcelona, pp. 169-187.

- (1987): El modelo de investigación-acción en el currículum. En SARRAMONA LÓPEZ, J. (Ed.): Curriculum y educación. Ceac. Barcelona, pp. 74-91.

- (1989): ¿Para qué la escuela en la revolución postindustrial. Identidad y cambio en la educación de los años 90. En ESTEVE ZARAZAGA, J. M. (Ed.): Objetivos y contenidos de la educación para los años noventa. Universidad de Málaga, pp. 157-179.

- (1991): La pedagogía como ciencia cognitiva. Revista Española de Pedagogía, no 188 , $123-145$.

(C) Ediciones Universidad de Salamanca

Teor. educ. 11,1999 , pp. 13-52 
- (1993a): Inteligencia, tecnología y escuela en la sociedad postindustrial. En McCLINTOCK, R. O.; STREIBEL, M. J. y VÁZQUEZ GÓMEZ, G.: Comunicación, tecnología y diseños de instrucción: la construcción del conocimiento escolar y el uso de los ordenadores. Ministerio de Educación y Ciencia, Madrid, pp. 207-251.

- (1993b): Problemas del conocimiento en la edad postindustrial: ¿qué debe enseñarse en la escuela?. En McCLINTOCK, R. O.; STREIBEL, M. J. y VÁZQUEZ GÓMEZ, G.: Comunicación, tecnologia y diseños de instrucción: la construcción del conocimiento escolar y el uso de los ordenadores. Ministerio de Educación y Ciencia. Madrid, pp. 253-269.

- (1999): Filosofía y Antropología del Currículo. En ARGOS, J. y EZQUERRA, Ma . P.: Principios del curriculum. IV Jornadas de Teorias e Instituciones Educativas Contemporáneas. Servicio de Publicaciones de la Universidad de Cantabria. Santander, pp. 31-47.

VERA, J. (1999): Cultura, diseño curricular y aprendizaje significativo. En RODRÍGUEZ NEIRA, T. y OTROS (Coords.): Cambio educativo: presente y futuro. Servicio de Publicaciones de la Universidad de Oviedo. Oviedo, pp. 201-210.

WATZLAWICK, P. y KRIEG, P. (Comps.) (1994): El ojo del observador. Contribuciones al constructivismo. Gedisa. Barcelona.

WHEELER, D.K. (1976): El desarrollo del curriculum escolar. Santillana. Madrid.

ZABALZA, M.A. (1991): Diseño y desarrollo curricular. Narcea. Madrid. $4 . \underline{a}$ de. 